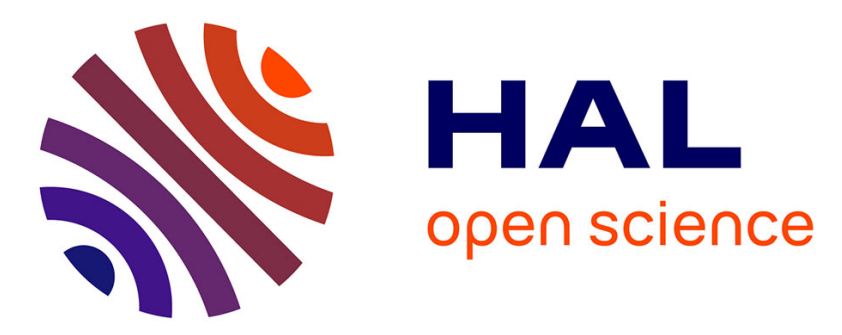

\title{
Experimental study of turbulent-jet wave packets and their acoustic efficiency
}

David E.S. Breakey, Peter Jordan, André Cavalieri, Petrônio Nogueira, Olivier Léon, Tim Colonius, Daniel Rodriguez

\section{- To cite this version:}

David E.S. Breakey, Peter Jordan, André Cavalieri, Petrônio Nogueira, Olivier Léon, et al.. Experimental study of turbulent-jet wave packets and their acoustic efficiency. Physical Review Fluids, 2017, 2 (12), 10.1103/PhysRevFluids.2.124601 . hal-02118994

\section{HAL Id: hal-02118994 \\ https://hal.science/hal-02118994}

Submitted on 3 May 2019

HAL is a multi-disciplinary open access archive for the deposit and dissemination of scientific research documents, whether they are published or not. The documents may come from teaching and research institutions in France or abroad, or from public or private research centers.
L'archive ouverte pluridisciplinaire HAL, est destinée au dépôt et à la diffusion de documents scientifiques de niveau recherche, publiés ou non, émanant des établissements d'enseignement et de recherche français ou étrangers, des laboratoires publics ou privés. 


\title{
Experimental study of turbulent-jet wave packets and their acoustic efficiency
}

\author{
David E. S. Breakey* \\ Department of Mechanical and Manufacturing Engineering, Trinity College Dublin, \\ College Green, Dublin 2, Ireland \\ Peter Jordan \\ Département Fluides, Thermique, Combustion, Institut Pprime, CNRS-Université de Poitiers-ENSMA, \\ 86036 Poitiers Cedex, Poitiers, France
}

André V. G. Cavalieri and Petrônio A. Nogueira

Divisão de Engenharia Aeronáutica, Instituto Tecnológico de Aeronáutica, 12228-900 São José dos Campos, São Paulo, Brazil

Olivier Léon

Department of Aerodynamics and Energetics Modelling, ONERA, Toulouse 31055, France

Tim Colonius

Division of Engineering and Applied Science, California Institute of Technology, Pasadena, California 91125, USA

Daniel Rodríguez

Laboratory of Theoretical and Applied Mechanics (LMTA), Graduate Program in Mechanical Engineering (PGMEC), Universidade Federal Fluminense (UFF), Niterói, Rio de Janeiro 24210-240, Brazil

(Received 15 June 2017; published 1 December 2017)

This paper details the statistical and time-resolved analysis of the relationship between the near-field pressure fluctuations of unforced, subsonic free jets $(0.4 \leqslant M \leqslant 0.6)$ and their far-field sound emissions. Near-field and far-field microphone measurements were taken on a conical array close to the jets and an azimuthal ring at $20^{\circ}$ to the jet axis, respectively. Recent velocity and pressure measurements indicate the presence of linear wave packets in the near field by closely matching predictions from the linear homogenous parabolized stability equations, but the agreement breaks down both beyond the end of the potential core and when considering higher order statistical moments, such as the two-point coherence. Proper orthogonal decomposition (POD), interpreted in terms of inhomogeneous linear models using the resolvent framework allows us to understand these discrepancies. A new technique is developed for projecting time-domain pressure measurements onto a statistically obtained POD basis, yielding the time-resolved activity of each POD mode and its correlation with the far field. A single POD mode, interpreted as an optimal high-gain structure that arises due to turbulent forcing, captures the salient near-field-far-field correlation signature; further, the signatures of the next two modes, understood as suboptimally forced structures, suggest that these POD modes represent higher order, acoustically important near-field behavior. An existing Green's-function-based technique is used to make far-field predictions, and results are interpreted in terms of POD/resolvent modes, indicating the acoustic importance of this higher order behavior. The technique is extended to provide time-domain far-field predictions.

DOI: 10.1103/PhysRevFluids.2.124601

\footnotetext{
*Present address: Department of Chemical and Materials Engineering, University of Alberta, Edmonton, AB T6G 1H9, Canada; breakeyd@tcd.ie
} 


\section{INTRODUCTION}

\section{A. Background}

The analysis of noise generation from turbulence in a jet is a story of two different complexities. In the turbulent flow region, the fluctuations satisfy the full nonlinear Navier-Stokes equations, numerical solutions of which are now possible in simple cases but not necessarily practical or informative for jet design. In the far field, the pressure fluctuations follow simple linear acoustics equations, which can be easily solved for given boundary conditions (typically a pressure distribution on a surface surrounding the turbulent flow). This reduced complexity of the far field gives hope to researchers that the acoustically important behavior of jet turbulence can also be captured with a reduced-order model of the jet dynamics [1]. This hope was tantalized by the discovery of coherent structures in turbulence in the early 1960s, a development from the earlier understanding of turbulence as a superposition of stochastic eddies. The role of coherent structures in turbulence and noise production has been a topic of intense research ever since. A candidate source structure that is consistent with many of the observed features of jet noise is an axially extended wave packet [2]. Forerunners such as Mollo-Christensen [3,4], Crow and Champagne [5], and Crighton [6] made early observations of these signatures and developed the mathematical basis for their sound production and prediction. In all likelihood, wave packets will not tell the whole story of jet noise emissions, but they do appear to account for many of the salient features, especially at low emission angles. Jordan and Colonius [1] have presented a comprehensive review of the various works dealing with wave packets and jet noise sources.

Crow [7] (see Ref. [6]) was the first to show that the sound emissions from simple wave-packet sources could be calculated analytically, and more recent work has noted the effect on sound emissions of several wave-packet parameters including the spatial envelope [8], temporal growth and decay [9], and spatiotemporal jitter [10], including its statistical signature: coherence decay [11]. Early experimental work in analyzing wave packets focused on forced jets because measurements could be phase locked to an external trigger, but recent work has also found evidence of wave packets in the velocity fields of natural jets [12], and it has furthermore been shown that such wave packets in the flow can be educed using flow-acoustic correlations [13].

The physical basis for the appearance of wave packets in a jet arises from a stability analysis of the mean-flow profile [3,4]. Early stability analyses were performed in a locally parallel framework [14]; these were followed by weakly nonparallel [15-17] and, more recently, fully global calculations [18-20]. The use of the time-averaged mean as an equivalent laminar base flow [15] has generally been justified by appealing to a scale and energy separation between wave packets and turbulence, the effect of the latter being limited to its role in generating the mean flow. In a recent study by Beneddine et al. [21], where the problem is considered by analysis of the resolvent of the linear operator, it is argued that under certain conditions (for weakly nonparallel, convectively unstable flows, when the first singular value of the resolvent largely dominates those of the suboptimals) the mean-flow stability approach can be more rigourously justified. The argument is based on the observation that, under these conditions, the coherent flow response, which results from a driving of the linear operator by nonlinear turbulence interactions that take the form of a generalized, frequency-dependent Reynolds stress [22], may be provided by a rank-1 model (the linear operator driven by a single forcing mode). A second observation, that the spatial support of the generalized Reynolds stress (the forcing mode), for flows respecting the aforesaid conditions, tends to be localized at the upstream boundary, is the basis of an argument that a homogeneous linear stability problem forced at the inflow is equivalent to the more general inhomogeneous model; i.e., introduction of a spatially localized, generalized Reynolds stress at the inflow is sufficient to correctly compute the coherent flow response.

It has been found, however, in a number of recent resolvent analyses of high-Reynolds-number turbulent jets, that these flows do not fulfill some of these conditions. For low frequencies ( $\mathrm{St} \leqslant 0.3$ ), the first singular value of the axisymmetric mode has a similar order of magnitude as those of the suboptimals, and the optimal forcing mode is spatially extended throughout the flow. At higher 
frequencies, where a gain separation does exist, it is found that the flow response (obtained using proper orthogonal decomposition in the frequency domain) is not very well reproduced by either solutions of the homogeneous linear problem, or singular modes of the resolvent. Indeed, good agreement is only observed between the stability predictions and the second-order, single-point statistical moments upstream of the end of the potential core. In the downstream region, on the other hand, recent studies support the idea that wave packets persist here, but are forced by turbulence [23-25]. Despite the low levels of the forcing modes in this region, stabilization of the dominant convective instability leads to an accrued sensitivity to nonlinear effects that act predominantly via the critical layer. The same studies also suggest that such turbulence forcing is necessary to explain higher order statistical wave-packet moments, such as the two-point coherence. These higher order features cannot be captured by a rank-1 resolvent model, however: At least two modes are necessary. The linearized Navier-Stokes equations, in either homogeneous or inhomogeneous forms (the former with inhomogeneous boundary conditions modeling disturbances on the nozzle exit plane, the latter considering background turbulence forcing coherent responses), can thus provide a basis by which to study and understand wave packets in turbulent jets, and on which to build noise-prediction schemes. The stability predictions in this work are calculated by solution of the parabolized stability equations (PSE) [16] and the results are compared with experimental measurements.

\section{B. Factors affecting wave-packet emissions}

The pertinent parameters for describing a wave packet are the wave number, $k$; angular frequency, $\omega$; the spatial amplitude envelope, characterized by an amplitude length scale, $L$; and a spatial coherence envelope, characterized by a coherence length scale, $L_{c}$. Specifically, $L$ and $L_{c}$ are the characteristic lengths of the decay of the wave-packet amplitude and coherence, respectively. Physically, this is defined as the distance at which the envelopes decay to $1 / e$ times the maximum value. For example, for source models, if $x$ and $x_{2}$ are two axial locations in the jet, the amplitude and coherence envelopes would be described as $\exp \left(-x^{2} / L^{2}-x_{2}^{2} / L^{2}\right)$ and $\exp \left[-\left(x-x_{2}\right)^{2} / L_{c}^{2}\right]$, respectively (see Ref. [11]). For context, in the case of the present $M=0.6$ data, for $\mathrm{St}=0.4$, we observed $L \approx 1.5 D$ and $L_{c} \approx 1.7 D$; and for $\mathrm{St}=0.6$, we observed $L \approx 1.3 D$ and $L_{c} \approx 1.4 D$.

Together, $k$ and $\omega$ prescribe the wave packet's convection speed, $U_{c}=\omega / k$, which can be subsonic $\left(U_{c}<c_{0}\right)$, sonic $\left(U_{c}=c_{0}\right)$, or supersonic $\left(U_{c}>c_{0}\right)$ relative to the ambient speed of sound, $c_{0}$, depending on the jet's convective Mach number $M_{c}=U_{c} / c_{0} . M_{c}, L$, and $L_{c}$ are key parameters in determining the efficiency of the conversion of wave-packet fluctuations into propagating sound emissions. This is because only the part of a wave packet's $k-f$ spectrum that is supersonic radiates to the far field $[1,8]$. A subsonic convected wave with no amplitude modulation is silent, while the same wave convecting supersonically has strong Mach-wave emissions. A subsonic wave packet of given spatial amplitude envelope, but no spatial coherence envelope $\left(L_{c}=\infty\right)$, generates several orders of magnitude less sound energy than a wave packet of similar amplitude envelope but with a realistic spatial coherence envelope ( $L_{c}$ and $L$ with comparable magnitudes). In order for a subsonic wave packet to emit sound at all, it must have a finite spatial envelope, which causes part of the $k-f$ spectrum to "leak" into the radiating zone, resulting in escaping sound emissions [1]; the coherence envelope further enhances this effect $[11,26]$.

The average spatial amplitude envelope is determined by the wave packet's amplification, stabilization, and decay as it convects in the jet. This is the envelope that is predicted by a stability analysis and that can be extracted empirically by a proper orthogonal decomposition (POD [17,27]). The particular shape of the average wave packet influences the portion of the fluctuations that radiates and therefore the acoustic efficiency of the wave packet.

We will show that the spatial coherence envelope is underpinned by the higher order POD modes, which are, in turn, related to the suboptimal response modes of a resolvent analysis- the appropriate stability framework for consideration of forced wave packets [24,25,28]. The precise relationship between these suboptimal modes and their stochastic POD counterparts, and the manner in which they determine the coherence decay is presently the object of a number of studies. The coherence 
DAVID E. S. BREAKEY et al.
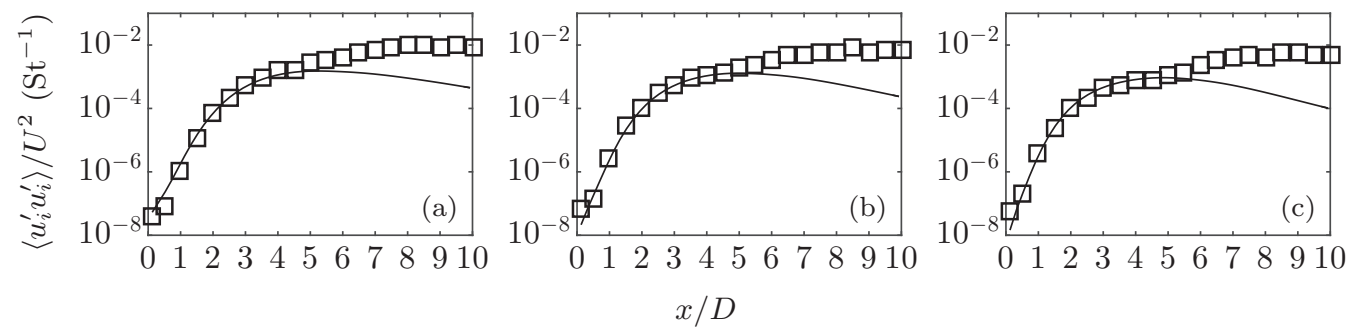

FIG. 1. Comparison between linear PSE predictions (-) and measured $u^{\prime}(\square)$ for the axisymmetric mode on the jet centreline for $M=0.4$ and $0.4 \leqslant \mathrm{St} \leqslant 0.6$. Measured and PSE data from Cavalieri et al. [12]. (a) $\mathrm{St}=0.4$, (b) $\mathrm{St}=0.5$, and (c) $\mathrm{St}=0.6$.

decay, a statistical, frequency-domain measure, is driven by space-time wave-packet modulation, modeled, for instance, by Sandham et al. [9] and Cavalieri et al. [10]. The possibility of such time-domain effects is considered in the analysis in this paper, and a link is established with POD modes, coherence decay, and acoustic efficiency.

\section{Motivation for current experiments}

Despite recent favorable agreement demonstrated between PSE predictions and both in-flow and near-field flow fluctuations [12,17], as well as wave-packet predictions and far-field pressure statistics [2,29], several questions remain unanswered.

A convincing explanation has not been found for the discrepancy between measurement and solutions of the homogeneous linear model in the region downstream of the end of the potential core, a discrepancy discussed above and illustrated in Figs. 1 and 2 for the same jets studied here. Also, a clear relationship has not yet been determined between time-domain wave-packet fluctuations and far-field sound emissions. To this end, the objectives of the paper are (1) to determine if the fluctuations in the region downstream of the jet can be represented by POD and stability theory; (2) to investigate the relationship between reduced-order wave-packet representations of the near-field and the observed far-field sound emissions; and (3) to experimentally interpret the issue of coherence decay and acoustic efficiency [11], particularly with respect to low-order wave-packet representations.

The paper reports the analysis of data obtained from a ring array of microphones in the jet far field and by a conical array of microphones in the part of the near pressure field of the jet labeled the linear hydrodynamic region [30]. In this region, the pressure fluctuations are dominated by waves with subsonic phase speed in the axial direction. These fluctuations are thus mostly of hydrodynamic nature, but they do not exhibit the rotational, turbulent nature of the fluctuations within the jet flow.
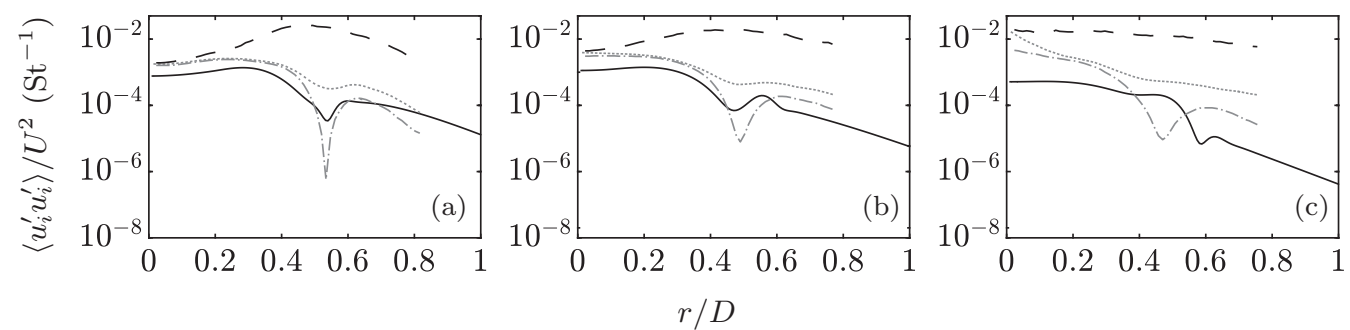

FIG. 2. Comparison between linear PSE predictions and measured $u^{\prime}$ for $M=0.4$ at three axial stations. , PSE; $-\ldots$, experimental $u^{\prime}$; , axisymmetric mode of $u^{\prime}$; , first POD mode of axisymmetric mode of $u^{\prime}$. Data from Cavalieri et al. [12]. (a) $x / D=3.5$, (b) $x / D=5$, and (c) $x / D=8$. 
The near-field array used in these experiments is similar to those used by Suzuki and Colonius [30] and Gudmundsson and Colonius [17] in their similar investigations.

An interesting aspect of this work is the simultaneous measurement of the far-field pressure, enabling analysis of the causal link between the near-field wave packets and the emitted sound. In this case, the near-field-far-field correlation results will give strong evidence to back up the hypothesis that the suboptimal modes can be considered in concert with the optimal mode to indicate that low-order behavior explains much of the far-field emissions; however, it will be clear that single POD modes will not be enough to represent this behavior. A Green's-function-based technique [29,31] is used to project the near-field representations to the far field, giving evidence of the efficiency of these low-order representations.

The rest of this paper is organized as follows: Sec. II outlines the PSE approach to wave-packet analysis in jets, Sec. III outlines the experimental setup used in this work, Sec. IV presents the analysis of the POD modes, while Sec. V covers the time signal projection onto a reduced-order basis, near-field-far-field correlation, and far-field prediction techniques. Particular attention is paid to justifying the resolvent discussion with the current results, but no resolvent computations are performed here. Section VI outlines the implications of this work and future avenues to be pursued.

\section{PARABOlized STABILITY EQUATIONS}

PSE provides a computationally efficient tool to model wave packets. Herbert [16] has given a full description of the development and application of PSE, and recent developments in the application of PSE to the near-field statistics of jet flows have been described by Gudmundsson and Colonius [17]. In addition, the PSE computations used in this paper for comparison to the experimental results-which are limited to linear PSE-were already presented by Cavalieri et al. [12]. This section therefore includes only the level of detail necessary to motivate the current work.

The stability computation begins with either a measured or calculated mean flow profile. For design purposes, this profile could even be calculated by a Reynolds-averaged Navier-Stokes simulation. The two-dimensional eigenvalue problem associated with the jet mean flow can nowadays be tackled directly with large computational expense [18], but for expediency and to draw closer connections with past work, we rely on the slowly varying approximation as approximated by PSE. For jet calculations, a PSE solution is obtained by taking the parallel linear stability solution at the nozzle exit as a boundary condition and then marching the solution downstream, frequency by frequency, to solve for the shape functions throughout the whole jet flow. This is possible due to the approximate parabolization of the equations, with each step independent of the solution downstream. The outputs of the stability analysis are the amplitudes and relative phases of the flow variable fluctuations in the jet $\left(u^{\prime}, v^{\prime}, w^{\prime}, p^{\prime}, \rho^{\prime}, T^{\prime}\right)$ for a given frequency and azimuthal mode.

The PSE technique cannot directly resolve acoustic radiation at subsonic speeds because there is a mismatch between the wavelength of the instability wave and the wavelength of the acoustic wave at the same frequency. In PSE, these waves are damped at a rate that is proportional to the difference in wavelength (and thus inversely proportional to the jet Mach number) and this leads to a more rapid decay of the PSE shape functions than would be obtained by a full solution of the linear equations [24]. As we discuss in Sec. IV, this inherent acoustic damping of PSE can lead to discrepancies between the inferred near-field amplitude of wave packets when using microphones placed outside the jet shear layer.

In the linear framework, each mode amplitude includes a free constant that must be chosen after comparison with one of the flow variables. With the present jets, these constants have already been obtained by comparison with $u^{\prime}$ measured on the jet centerline [12]. PSE predicts fluctuations in the jet that are coherent over a large axial extent. In regions of the jet dominated by small-scale turbulence, the axially coherent fluctuations may be masked by these uncorrelated fluctuations. To 


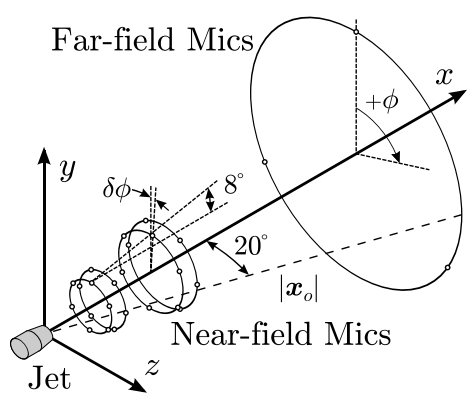

(a)

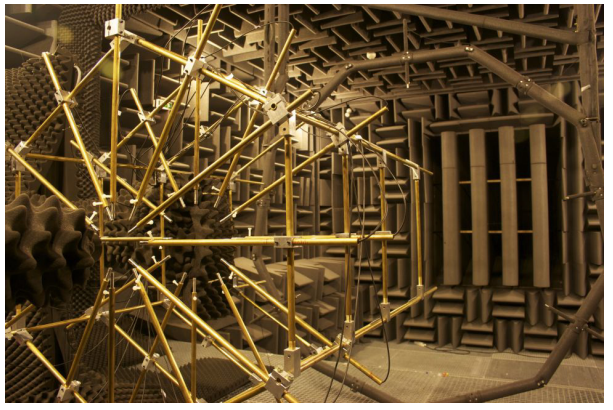

(b)

FIG. 3. Overview of four-ring setup. (a) Overview and (b) Installed rig.

compare PSE to the axially coherent fluctuations in the jet, POD is typically used to isolate the most energetic coherent fluctuations (i.e., the lowest POD modes).

\section{EXPERIMENTAL SETUP}

The experiments were carried out in an anechoic free jet facility with a cutoff frequency of $200 \mathrm{~Hz}$ at the Centre d'études aérodynamiques et thermiques (CEAT), Institut Pprime, Poitiers, France. Two experimental campaigns obtained measurements in the pressure field generated by a jet with nozzle diameter $D=50 \mathrm{~mm}$ and Mach numbers in the range $0.4 \leqslant M \leqslant 0.6$. The corresponding Reynolds number range $\operatorname{Re}=\rho_{0} U D / \mu$ was $4.2 \times 10^{5}$ to $5.7 \times 10^{5}$, where $U$ is the mean jet velocity at the center of the nozzle exit, $\rho_{0}$ is the density, and $\mu$ is the air viscosity measured at the nozzle exit. The temperature of the jets was kept at $20^{\circ} \mathrm{C}$. A carborundum trip placed $2.7 \mathrm{D}$ upstream of the nozzle lip ensured the boundary layer was turbulent at the jet exit. Both the acoustic far field [2] and the velocity field [12] of these jets have been previously examined, and more details are available in the cited papers. The lengths of the potential cores of the jets ranged between $5 \leqslant x / D \leqslant 5.5$.

In both of the current campaigns, far-field pressure measurements were recorded by an azimuthal ring array of three equispaced $1 / 4^{\prime \prime}$ GRAS $40 \mathrm{BP}$ microphones at $\theta=20^{\circ}$ and $\left|\vec{x}_{o}\right| / D=47.1$. The far-field array was limited to three microphones because the sound field of these jets at $\theta=20^{\circ}$ has been shown to be dominated by fluctuations in the axisymmetric mode $(m=0,[2])$, making resolution of higher azimuthal modes unnecessary for the Strouhal number $(\mathrm{St}=f D / U)$ range investigated here. The near-field array was made up of a number of azimuthal ring arrays. Azimuthal arrays were used to enable decomposition of the pressure signals into azimuthal Fourier modes, enabling azimuthal mode-by-mode analysis consistent with the azimuthal mode-by-mode calculation of the linear PSE solutions. An overview of the test setup is illustrated in Fig. 3 for one of the campaigns, and the experimental conditions for both campaigns are tabulated in Table I.

The focus of the first campaign was a simultaneous measurement of as much of the near field as possible to enable a time-domain comparison to the near-field fluctuations and the far-field sound. To that end, the near-field array comprised seven azimuthal rings, each with six microphones

TABLE I. Experiment conditions for both experimental campaigns.

\begin{tabular}{lllccc}
\hline \hline Campaign & $X / D$ & $X_{\max } / D$ & $r_{A} / D$ & $\Delta X / D$ & $M$ \\
\hline \multirow{3}{*}{ Seven ring } & 1.25 & 5.75 & 0.8 & & \\
\multirow{2}{*}{ Four ring } & 2 & 6.5 & 0.7 & 0.75 & $0.4,0.45, \ldots, 0.6$ \\
\hline \hline
\end{tabular}




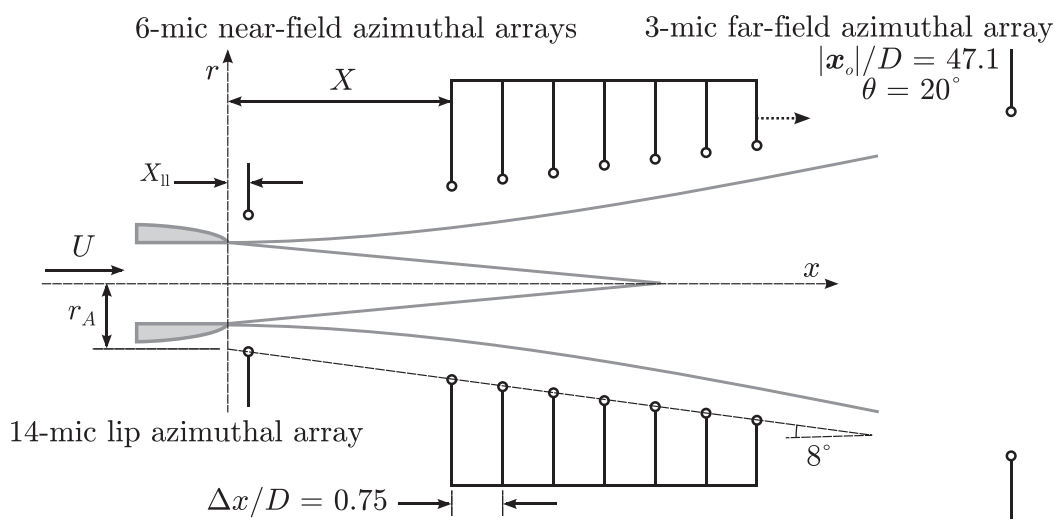

FIG. 4. Seven-ring near-field azimuthal array setup (7-pt correlation).

distributed azimuthally. A schematic of the setup is given in Fig. 4. There were a total of 42 microphones in the near-field cone. Two types of microphones were used in the near-field array because there was a limited number of high-quality precision microphones available. The first three rings used inexpensive $1 / 4^{\prime \prime}$ electret microphones, while the last four rings used $1 / 4^{\prime \prime}$ GRAS 40BP precision microphones. The frequency response of the electrets was found to closely match the GRAS microphones up to about $9 \mathrm{kHz}$ (St $\approx 2.2$ for $M=0.6$ ). The half-angle of the cone, $\alpha=8^{\circ}$, matched the expansion of the jet. The conical surface intersected the jet nozzle plane at a radial distance labeled $r_{A}$ (illustrated in Fig. 4), which was set between 0.6 and $0.8 D$ depending on the test configuration. Unless otherwise noted, the results presented below are for the $r_{A} / D=0.8$ case. The entire array was mounted on a traverse, which allowed the rings to span different portions of the jet near field ranging from $X$ to $X_{\max }$. In this campaign, the radii of the rings were not adjusted when $X$ was changed, meaning that each $X$ corresponded to a different $r_{A}$ as well as different axial coverage of the jet. Since the axial spacing was $0.75 D$, shifting $X$ in multiples of this amount gave measurements at the same axial locations on different conical surfaces, allowing a partial evaluation of the radial decay of the near-field fluctuations. At each axial position, measurements were obtained in the velocity range $0.4 \leqslant M \leqslant 0.6$ in increments of 0.05 . An additional ring of 14 microphones was included close to the nozzle lip in order to investigate the relationship between near-nozzle fluctuations and downstream activity. These data have not been examined here.

The second campaign, illustrated in Fig. 5, was designed to provide finer resolution measurement of the statistics of the near-field fluctuations. This array consisted of four rings of six $1 / 4^{\prime \prime}$ GRAS 40BP microphones that could be moved independently in the axial direction. This setup does not

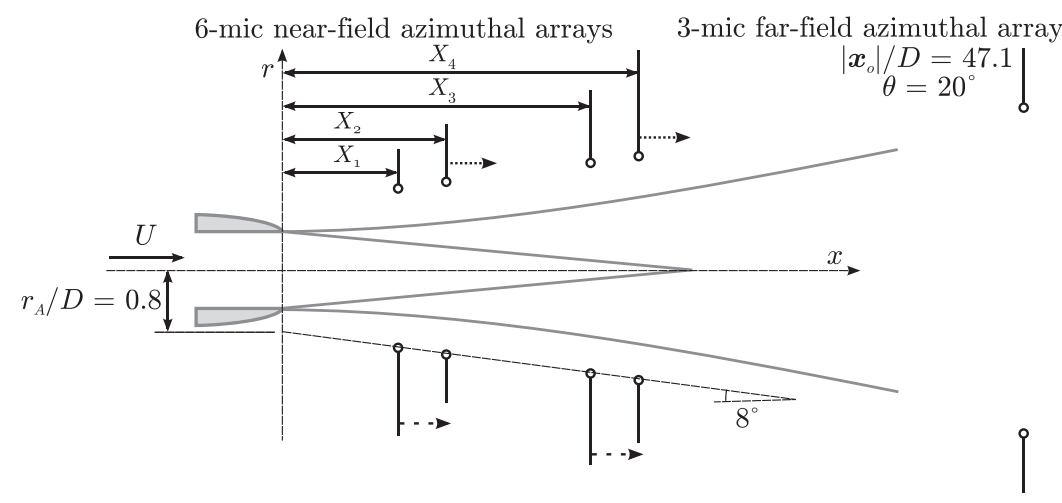

FIG. 5. Four-ring near-field azimuthal array setup (2-pt correlation). 
allow the monitoring of the space-time structure of the near-field pressure, but it does allow the statistical shape and energy of the modes to be determined via two-point correlations, allowing the construction of the cross-spectral matrix (CSM) describing the jet statistics. To ensure that all the measurements fell on the same conical surface $\left(r_{A} / D=0.8\right)$, the radial positions of the microphones were adjusted every time a ring was moved axially. The use of four rings-instead of the minimum two-simply reduced the number of acquisitions required to span the whole jet length. The final axial resolution of the measurements was $0.4 D$, and the measurements were made in the axial range $0.5 \leqslant x / D \leqslant 8.9$ (22 measurement locations) for $M=0.6$. Again, these parameters are summarized in Table I. All microphone measurements for both campaigns were recorded at $f_{s}=100 \mathrm{kHz}$ with a sample time of $10 \mathrm{~s}$.

\section{ANALYSIS OF NEAR-FIELD PRESSURE}

\section{A. POD mode analysis}

First, the pressure signals are decomposed by Fourier series into azimuthal modes and frequency components as

$$
\begin{aligned}
& \tilde{p}_{m}(x, t)=\tilde{p}(x, m, t)=\int p^{\prime}(x, \phi, t) e^{\mathrm{i} m \phi} d \phi, \\
& P_{m, \omega}(x)=P(x, m, \omega)=\int \tilde{p}_{m}(x, t) e^{-\mathrm{i} \omega t} d t,
\end{aligned}
$$

where $\omega$ is the angular frequency. Again, for the present results, only the axisymmetric mode $(m=0)$, which has been shown to dominate the low-angle emissions (2; see also Sec. IC), is considered. The two-point CSM, $\mathrm{R}_{m, \omega}\left(x, x_{2}\right)$, is then formed as

$$
\mathrm{R}_{m, \omega}\left(x, x_{2}\right)=\left\langle P_{m, \omega}(x) P_{m, \omega}^{*}\left(x_{2}\right)\right\rangle,
$$

where * indicates complex conjugation and \langle\rangle is the ensemble average, so that the POD problem on the cone surface is represented by the Fredholm integral equation

$$
\frac{2 \pi}{\cos \alpha} \int \mathrm{R}_{m, \omega}\left(x, x_{2}, r, r_{2}\right) \xi_{m, \omega}\left(x_{2}, r_{2}\right) r_{2} d x_{2}=\lambda_{m, \omega} \xi_{m, \omega}(x, r)
$$

with $r=\left(x-X_{0}\right) \tan \alpha$, where $X_{0}$ is the virtual origin of the antenna array and $\alpha$ is the cone half-angle. $\lambda_{m, \omega}^{(i)}$ and $\xi_{m, \omega}^{(i)}(x)$ are the eigenvalues and eigenvectors of the matrix $\mathrm{R}_{m, \omega}\left(x, x_{2}\right)$, respectively. The kernel $r_{2} \mathrm{R}_{m, \omega}\left(x, x_{2}, r, r_{2}\right)$ is not Hermitian, but following Jung et al. [32] and Cavalieri et al. [12], it can be made Hermitian by multiplying Eq. (4) by $\sqrt{r}$ and considering the kernel $\sqrt{r r_{2}} \mathrm{R}_{m, \omega}\left(x, x_{2}, r, r_{2}\right)$ with the eigenvector $\sqrt{r_{2}} \xi_{m, \omega}^{(i)}(x)$. The constant outside the integral can also be absorbed into the eigenvalues. Now that the kernel is Hermitian and positive semi-definite, the eigenvalues are real and greater than or equal to zero while the eigenvectors are complex-valued. In practice, small errors in the CSM can result in some negative eigenvalues, which are considered to be zero. In the discrete case, the matrix has dimensions $I \times I$, where $I$ is the number of axial observation locations. The calculation is performed separately for each combination of frequency and azimuthal mode. An individual POD mode is obtained by

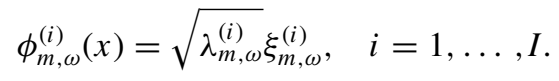

By construction, each set $\left\{\xi_{m, \omega}^{(i)}(x), i=1, \ldots, I\right\}$ forms a complete basis for $\tilde{p}_{m}(x, t)$, but including only those modes for which $\lambda_{m, \omega}^{(i)}>0$ has the added advantage of reducing the span of the basis to the span of the realizations that were used to create the eigenvectors [33], forcing all relevant boundary and physical constraints to be met automatically in any reconstruction.

The Fourier analysis in $t$ [Eqs. (2) and (3)] was performed in blocks of $N=244$ points, corresponding to a Strouhal step size $(\Delta \mathrm{St})$ of 0.1 , which is the same as the PSE computations. No windowing was used in the calculation. Since each recording had one million points $(10 \mathrm{~s}$ at 


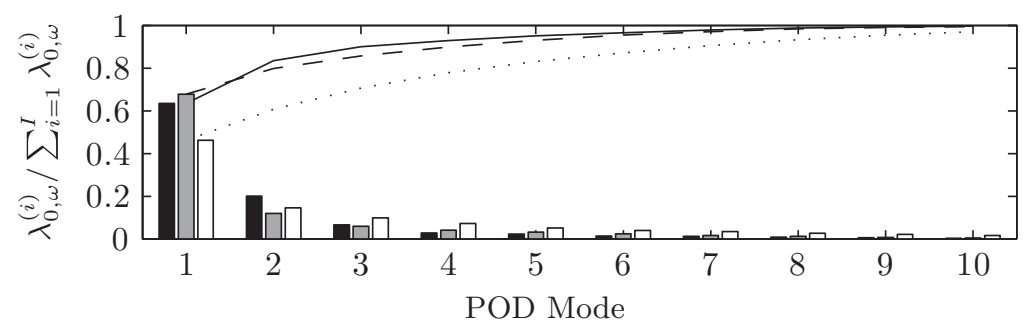

FIG. 6. Energy distribution (bars) and cumulative sum (lines) for the first 10 POD modes for $m=0$ from the four-ring array measurements. $\mathrm{St}=0.2$ (black bars and solid line), $\mathrm{St}=0.5$ (gray bars and dashed line), and $\mathrm{St}=0.8$ (white bars and dotted line).

$100 \mathrm{kHz}$ ), this gave $\approx 4500$ blocks with $10 \%$ overlap, ensuring that the CSM and POD modes were temporally converged.

Figure 6 shows the energy distribution of the various POD modes at selected frequencies obtained with the high-resolution four-ring array measurements. Most of the energy of the axisymmetric mode $(45-65 \%)$ is captured in its first POD mode, and this proportion is lowest for higher St. This first POD mode is the focus of the comparison with PSE, but higher order POD modes are also considered in the correlation results of Secs. V A and VB, as well as in the higher order interpretation of the amplitude and coherence envelopes (Secs. IV C and IV E, respectively).

\section{B. Note on the physical interpretation of POD modes}

POD has been used in fluid mechanics since its introduction by Lumley [34]. It is an empirical decomposition-it finds modes specific to the unsteady data considered. While this can be advantageous in so far as the modal decomposition is "customized" to perform an optimal compression for the phenomena under study, it can make physical interpretation difficult. For instance, it is not straightforward to associate modal structures to specific physical phenomena, or to relate modes to the equations of motion.

Farrell and Ioannou [35] have proposed a framework that permits the second point above to be addressed, linking time-domain POD modes to the equations of motion, and in doing so providing a link between stochastic and deterministic frameworks; these links have more recently been explored by Dergham et al. [36] and by Semeraro et al. [25], who reworked the analysis of Farrell and Ioannou [35] in the frequency domain. The approach involves considering the linearized equations of motion subject to stochastic "extrinsic" forcing, which would be supplied by background turbulence. As discussed in the introduction, resolvent analysis constitutes the appropriate framework for such a system. The central result of Farrell and Ioannou [35] is that if a linear system is subjected to uncorrelated space-time forcing (white noise), the time-domain POD modes that are computed using the stochastic data generated in response to the forcing can also be obtained as eigenmodes of a solution to a Lyapunov equation involving the linear operator. In addition to this, the frequencydomain POD modes of a linear system forced with white noise are identical to the response modes of the resolvent associated with the linear operator [25]. Such eigenmodes are thus deterministic, depending only on the structure of the linear operator. The framework proposed by Farrell and Ioannou [35] thus provides a connection between the stochastic and deterministic worlds.

In the framework of stability analyses, POD has previously been used as a means by which to filter measured data in order to separate "coherent" from "incoherent" motions, the former being considered as synonymous with wave packets [17], also termed the "spectral mode of the flow" by Beneddine et al. [21]. But the large quantitative acoustic inaccuracy of homogeneous linear models, and rank-1 POD models, is an indication that the higher order degrees of freedom, that can be accounted for by using a larger number of POD modes, need also to be incorporated in linear models, by means of an appropriate number of resolvent modes and associated generalized 

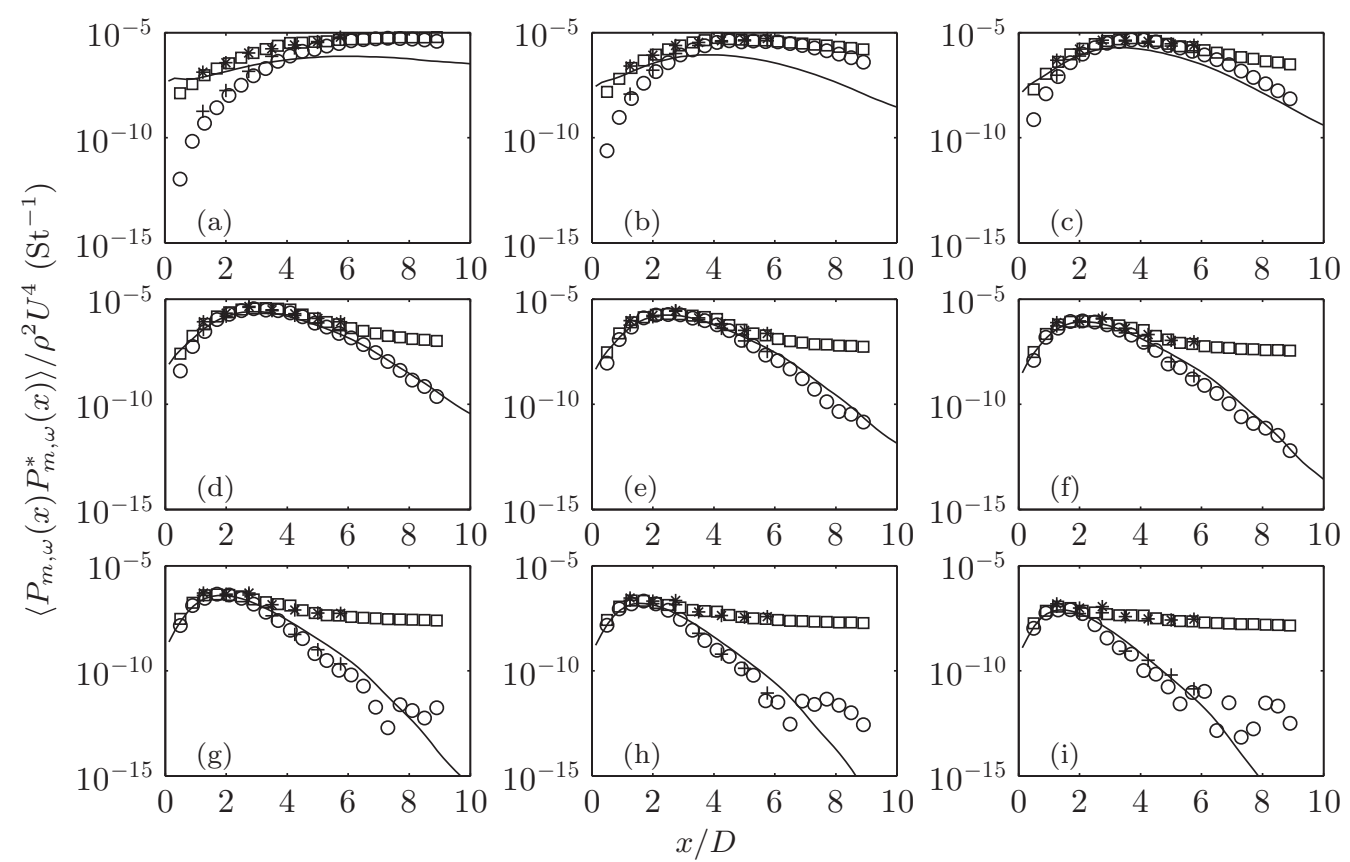

FIG. 7. Comparison of PSE estimates and measured autospectra magnitudes for $m=0$ and $M=0.6$. PSE; $\square$, four-ring full signal; $\bigcirc$, four-ring first POD mode; $*$, seven-ring full signal; + , seven-ring first POD mode. (PSE estimates multiplied by 10.) (a) $\mathrm{St}=0.1$, (b) $\mathrm{St}=0.2$, (c) $\mathrm{St}=0.3$, (d) $\mathrm{St}=0.4$, (e) $\mathrm{St}=0.5$, (f) $\mathrm{St}=0.6$, (g) $\mathrm{St}=0.7$, (h) $\mathrm{St}=0.8$, and (i) $\mathrm{St}=0.9$.

Reynolds stresses. At the same time, it should be noted that based on the current understanding of these dynamics, we do not expect that the simple case of white noise forcing would be sufficient to fully model jet turbulence. Instead, we expect that this quantitative inaccuracy points to the fact that it will be necessary to determine a more specific "colored" forcing that is responsible for the observed, more complex dynamics. Progress in determining this "color" has already begun [37], and a fuller understanding of it is a long-term goal of the current work.

In what follows, the coherent part of the near-field pressure will be assessed by means of POD, and we will be interested in particular in the extent to which low-rank representations are capable of correcting the discrepancies between the homogeneous linear model and the measurement. The comparison metrics are those important for sound generation, as discussed earlier.

\section{The amplitude envelope}

Comparing the amplitude envelope of the PSE predictions to the experimental predictions indicates whether the PSE model is able to capture the near-field flow dynamics. A close match to the POD result indicates that the coherent (unit-coherence) fluctuations are captured, while a mismatch between the PSE/POD and the experimental results indicates incoherent fluctuations dominate in that region.

Figures 7 and 8 compare the PSE predictions of the azimuthal mode with the measured amplitude and phases of both the original signal and the first POD mode for different frequencies. For the current computations, the number of points used in the discrete Fourier transform corresponded to $\Delta \mathrm{St}=0.1$, which was the same resolution as the PSE predictions, which are taken from Cavalieri et al. [12]. The data from the seven-ring array are also included to show that the same amplitudes and mode shapes are obtained for the coarser-resolution array. The reported amplitudes are scaled 

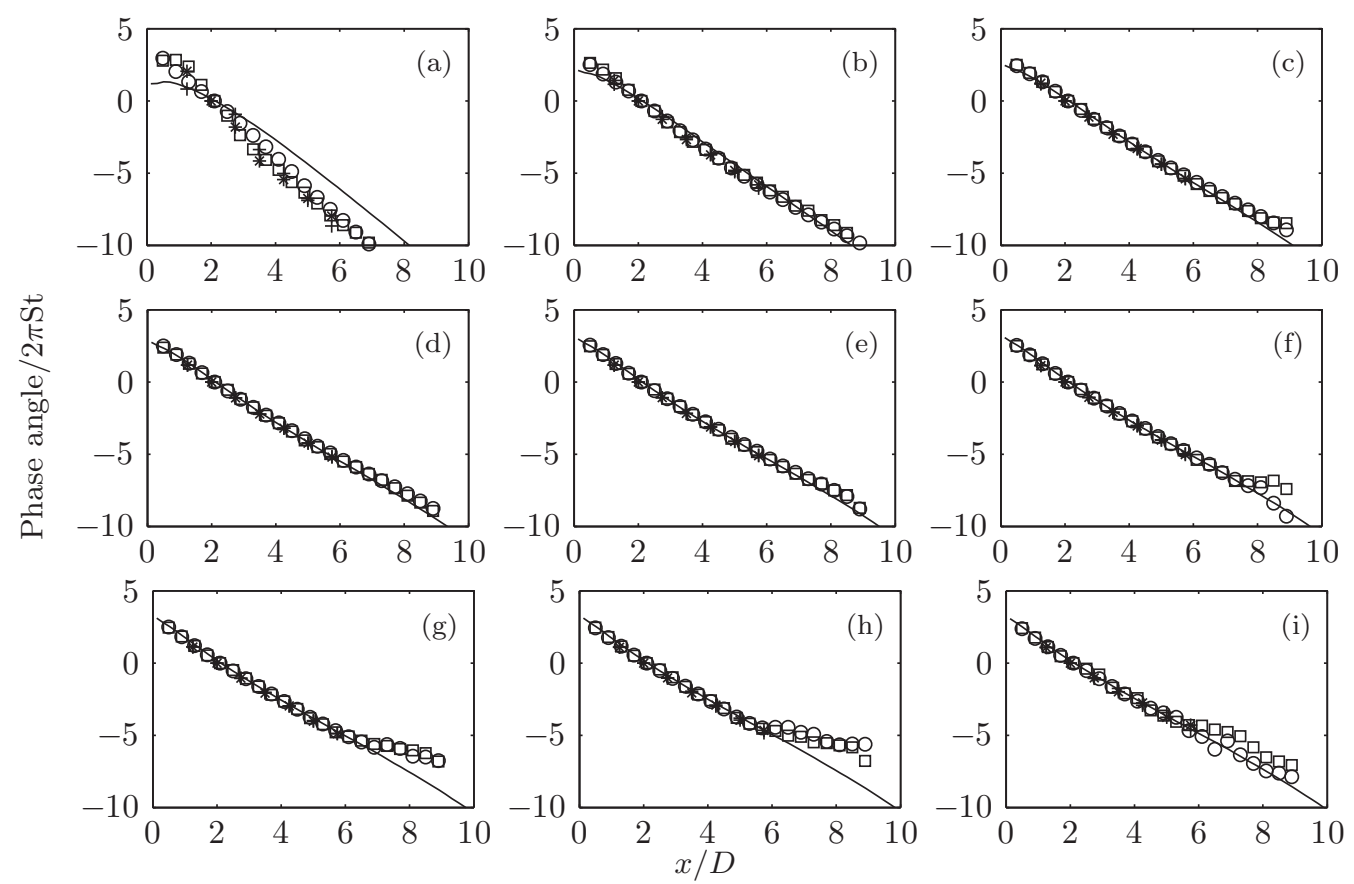

FIG. 8. Comparison of PSE estimates and measured autospectra phases for $m=0$ and $M=0.6$. Phase reference is $x / D=2$. See Fig. 7 for legend. (a) $\mathrm{St}=0.1$, (b) $\mathrm{St}=0.2$, (c) $\mathrm{St}=0.3$, (d) $\mathrm{St}=0.4$, (e) $\mathrm{St}=0.5$, (f) $\mathrm{St}=0.6$, (g) $\mathrm{St}=0.7$, (h) $\mathrm{St}=0.8$, and (i) $\mathrm{St}=0.9$.

estimates of $\left\langle P_{m, \omega}(x) P_{m, \omega}^{*}(x)\right\rangle$, and the phases are obtained using

$$
\text { Phase angle }=\arg \left(P_{m, \omega}(x)\right)-\arg \left(P_{m, \omega}\left(x_{\phi \mathrm{ref}}\right)\right),
$$

where the phase reference location is $x_{\phi \mathrm{ref}} / D=2$.

At each frequency, linear PSE predictions are scaled by a constant in order to compare them with the full and POD-filtered microphone measurements. This constant can in turn be inferred from the centerline hot-wire measurements of Cavalieri et al. [12]. However, we find that this leads to a poor agreement for the overall amplitude. For the plots in this section (all for $M=0.6$ ), we multiply the inferred amplitudes by an additional factor of 10 in order to compare the shape functions; the reasons for the discrepancy are explained in Sec. IV D.

In Fig. 7 for all cases, the PSE predictions beyond the end of the potential core underestimate the fluctuation intensity, but for St $\geqslant 0.3$ the first POD mode matches the PSE prediction nearly exactly. For $\mathrm{St} \geqslant 0.7$, this agreement breaks down at a distance well beyond the potential core length $(x / D \approx 5)$. The coarser seven-ring array also captures this behavior. For lower freqencies $(\mathrm{St} \leqslant 0.2)$, there is poor agreement in the amplitude shape and this is understood to be the result of PSE incorrectly modeling nonmodal effects that occur at low St. Ongoing work in resolvent analyses indicates that this is related to the dominance of nonmodal combinations of the Kelvin-Helmholtz instability with other stable modes, but further considering these details is beyond the scope of the present work. For the phase estimates in Fig. 8, the agreement is also striking for $\mathrm{St}>0.1$. Again, for $\mathrm{St} \geqslant 0.7$ there is a breakdown well beyond the potential core, but the agreement is otherwise excellent. The good agreement between the seven-ring and four-ring measurements in Fig. 8 also shows that the phase response of the electret microphones used in the first three near-field azimuthal rings is sufficient to provide accurate measurements. The implication of this downstream agreement is that the average linear wave packet is present in the downstream region; the implication of the mismatch with the complete data is that higher order wave-packet degrees of freedom are not 

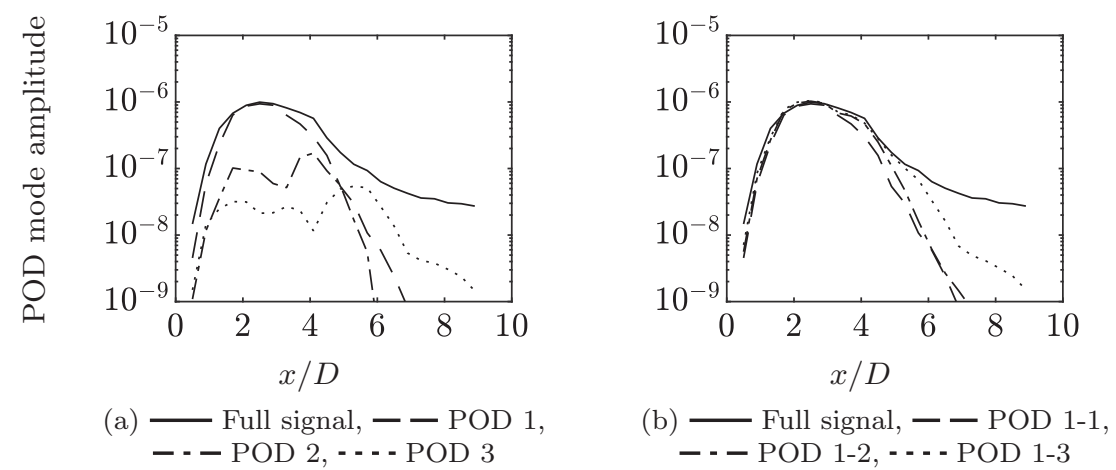

FIG. 9. Amplitude envelope of CSM reconstructions [see Eq. (5)] for $m=0, M=0.6$, and $\mathrm{St}=0.5$ from (a) individual and (b) cumulative POD modes.

correctly captured by the homogeneous linear-stability ansatz. We will later be interested in the question of the acoustic importance of this higher order wave-packet behavior.

Although a single POD mode leads to significant underestimations of the full power spectral density at downstream positions, as more information is incorporated into the reduced-order representation (reconstructing the CSM with multiple POD modes), the amplitude envelope shape improves. This is demonstrated in Fig. 9. The individual POD modes are presented in Fig. 9(a), showing that the higher modes begin to capture the downstream dynamics; and the cumulative POD modes (CSM reconstrutions) are presented in Fig. 9(b), showing that with a small number of POD modes (3 out of 22) an improvement of the representation of the downstream fluctuations is observed. These fluctuations have low order in the sense that they can be represented with a small number of degrees of freedom, but they are not coherent in the traditional sense (periodic). If they were periodic, they would be captured by a single POD mode. It could be said, for example, that their occurrence is intermittent but organized. However, the dynamics may also not be that simple. In line with the discussion of Sec. IV B, these three POD modes can be understood as the highest-gain structures that arise in response to stochastic forcing by turbulence nonlinearities. We can add that, as shown by Tissot et al. [23], the connection between the turbulent forcing and the high-gain structures occurs via the critical layer and involves an Orr-like mechanism affecting downstream fluctuations. The connection between these first few modes and the far field will be discussed in Sec. V B.

\section{Explanation for magnitude discrepancy in PSE estimates}

Initially it was thought that the scaling discrepancy mentioned in Sec. IV C between the PSE and experimental data was the result of a calibration or scaling error between the hot-wire measurements and the current pressure measurements, especially since the value of the multiplication factor required for a good match was 10 . However, careful inspection of both the original data and the data-processing procedures led to the conclusion that this was not the case, and further inspection of the available data was necessary.

As discussed in Sec. II, the approximate parabolization leading to the PSE equations damps acoustic waves relative to the primary wavelength of the near-field wave packet. To determine if this is the cause of the present discrepancy, we perform two checks on the data. First, the artificial damping of PSE should increase with radial distance from the jet, and second, the artificial damping should decrease as the jet Mach number is increased.

Since the seven-ring data are available at several radial locations, the PSE predictions, correctly scaled according the centerline hot-wire measurements, can be compared to the experimental results on the three conical surfaces $\left(r_{A} / D=0.6,0.7,0.8\right)$. We note that the first few POD modes from the seven-ring array are in good agreement with those from the four-ring array presented in the previous section (see Sec. V A). The comparison is made in Fig. 10 with no additional correction 

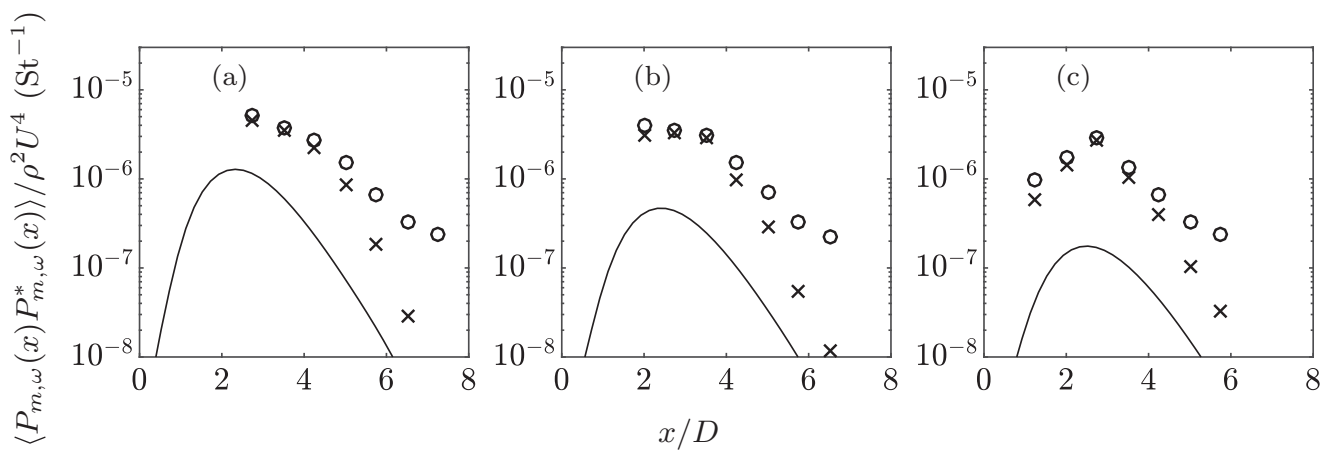

FIG. 10. Comparison of PSE estimates and measured autospectra magnitudes for $m=0, M=0.6$, and $\mathrm{St}=0.5$ for the seven-ring data at the three radial array locations. - , PSE; $\bigcirc$, full signal; $\times$, first POD mode. (PSE estimates not multiplied by 10.) (a) $r_{A} / D=0.8$, (b) $r_{A} / D=0.7$, and (c) $r_{A} / D=0.6$.

factor applied. The graphs show that as $r_{A}$ decreases, the gap between the experimental data and the PSE predictions decreases, consistent with the hypothesis that artificial acoustic damping inherent to PSE leads to the discrepancy. Though a factor of 10 gives a good fit for $r_{A} / D=0.8$ (Fig. 7), this factor is clearly not universal. This makes it unlikely that this factor is calibration or data-processing related, but rather a physical effect missing from the PSE model.

We therefore repeat the analysis for a jet at a higher Mach number of 0.9 and compare to POD modes educed from a well-validated large-eddy simulation (LES) of the same geometry [20,38]. The details are provided in Appendix A, where it is shown that the discrepancy between the inferred amplitudes is significantly reduced, again consistent with the hypothesis. Finally, we note that in applications to sufficiently supersonic jets, where there is no mismatch between the acoustic and wave-packet wavelengths at the Mach angle, PSE predictions can be extended directly into the acoustic field with reasonable agreement with the directly measured field from LES [39]. This effect, though not as pronounced at higher Mach number, clearly occurs in that dataset as well. Further comparison reveals that PSE overpredicts the radial decay rate of the wave-packet pressure fluctuations. This appears to be the result of the dominance of acoustic fluctuations with increasing $r$, fluctuations that are still in phase with the pressure wave packets and even have the same variation in magnitude in the axial direction.

Thus both analyses show that when using PSE, a discrepancy is expected between the wave-packet amplitude inferred from the jet centerline velocity fluctuations and microphones outside the jet shear layer. While no quantitative correction can be offered here, we note that a new technique, based on a rigorous parabolization of the compressible flow equations, has recently been proposed [40] that should alleviate the artificial acoustic damping inherent to PSE. We also note that while this discrepancy complicates the simplest interpretation of the data, it does not stand in the way of the conclusions drawn in this paper because it does not significantly affect the wave-packet shape.

In addition to these concerns, as noted in the introduction and as will be clear in the following sections, the key shortcoming of a rank-1 model such as PSE is that it does not capture the nonlinearities and turbulent forcing that have been shown to be necessary to capture higher order statistical wave-packet moments that are important to sound generation. For example, even a full linearized Euler equation solution of the near-field fluctuations has been shown to be insufficient to capture these sound-generating dynamics [41]. For these reasons, attempts to further improve the near-field representation have not been made here.

\section{E. The coherence envelope}

As has already been discussed, the shape of the coherence envelope is understood to be important in the acoustic emissions. A single POD mode always has unit coherence, so it cannot 


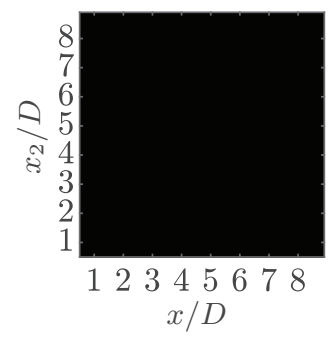

(a)

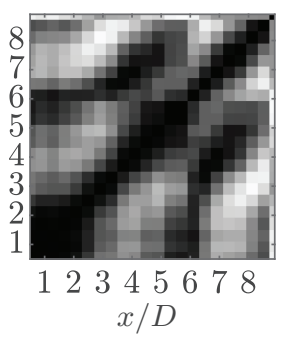

(b)

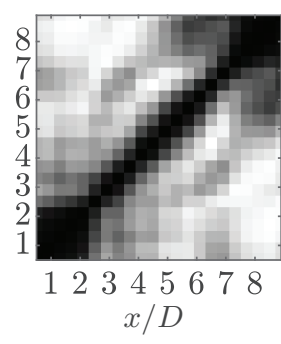

(c)

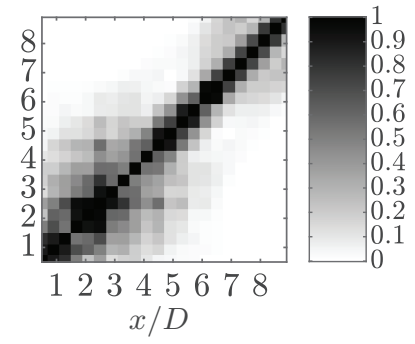

(d)

FIG. 11. Two-point coherence $\left(\left|\mathbf{R}_{m, \omega}\left(x, x_{2}\right)\right|^{2} / \mathbf{R}_{m, \omega}(x, x) \mathbf{R}_{m, \omega}\left(x_{2}, x_{2}\right)\right) m=0, M=0.6$, and $\mathrm{St}=0.5$ based on cumulative reconstructions using POD modes. (a) POD 1, (b) POD 1-2, (c) POD 1-3, and (d) Full signal.

capture higher order effects like jitter. The contrast between the experimental envelope and POD 1 envelope is demonstrated visually in Figs. 11(a) and 11(d), which show the two-point coherence $\left(\left|\mathrm{R}_{m, \omega}\left(x, x_{2}\right)\right|^{2} / \mathrm{R}_{m, \omega}(x, x) \mathrm{R}_{m, \omega}\left(x_{2}, x_{2}\right)\right)$ of the pressure fluctuations for $\mathrm{St}=0.5$ for the cumulative POD modes. In Figs. 11(b) and 11(c), $\mathrm{R}_{m, \omega}$ is taken as the cumulative reconstruction of the CSM using the first two or three POD modes, respectively. When $\mathrm{R}_{m, \omega}\left(x, x_{2}\right)$ is reconstructed from more than one POD mode (POD 1-2 or POD 1-3), the coherence envelope becomes more similar to that of the full signal. The fact that this requires only the first few POD modes as opposed to the full set of 22 (which would give the exact full signal cross-spectral matrix) is significant. It shows that a small number of degrees of freedom can capture the salient coherence features.

Given the discussion of Sec. IV B, we again see that, where coherence decay is concerned, to the empirical low-order description of the flow implied by the POD, there corresponds a low-order theoretical model. The fact that more than one POD mode is necessary to recreate this coherence decay can also explain why the far-field predictions (which will be presented in Sec. VC) using either the homogeneous linear model or a single POD mode are so poor.

\section{ANALYSIS OF NEAR-FIELD-FAR-FIELD RELATIONSHIP}

\section{A. Projection of time signals onto POD modes}

Since the POD modes form a complex-valued orthonormal basis for the measured pressure fluctuations [33], complex-valued coefficients of each POD mode, $b_{m, \omega}^{(i)}$, can be determined by projecting the Fourier-transformed time signals of the azimuthal coefficients, $P_{m, \omega}(x)$, onto the basis

$$
b_{m, \omega}^{(i)}=\int P_{m, \omega}(x) \xi_{m, \omega}^{(i) *}(x) d x .
$$

A low-order estimate, $\hat{\tilde{p}}_{m}(x, t)$, of the time-domain signal for an azimuthal mode $m$ can then be formed by the inverse Fourier transform as

$$
\hat{\tilde{p}}_{m}(x, t)=\frac{1}{2 \pi} \int\left[\sum_{i=1}^{J} b_{m, \omega}^{(i)} \xi_{m, \omega}^{(i)}(x)\right] e^{\mathrm{i} \omega t} d \omega,
$$

where $\hat{\tilde{p}}_{m}(x, t)=\tilde{p}_{m}(x, t)$ when $J=I$ or at least the number of $\lambda_{m, \omega}^{(i)}>0$. For discrete computations, Eqs. (7) and (8) can be applied sequentially to blocks of data where the number of points in each block should be the same as in Eq. (2). For consistency, when calculating ensemble averages with the reconstructed data, the blocks should be the same as in the reconstruction.

In principle, for a full time-domain reconstruction using a particular POD basis, it is necessary to have simultaneous measurements at all the original ring locations used for obtaining that basis. This means that a time-domain decomposition of all 22 POD modes available (per azimuthal mode) using the four-ring array is not possible, and only statistical information is available. However, since the 


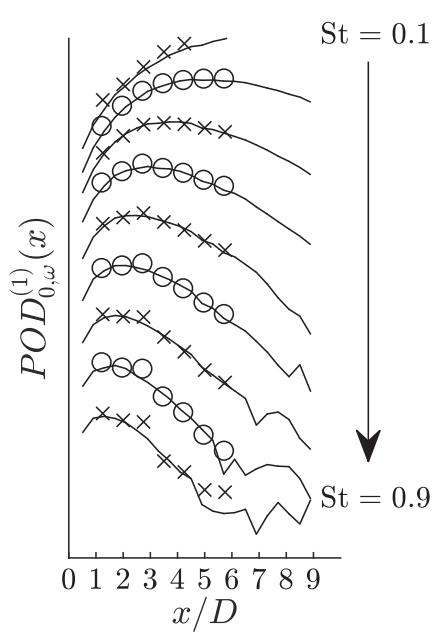

(a)

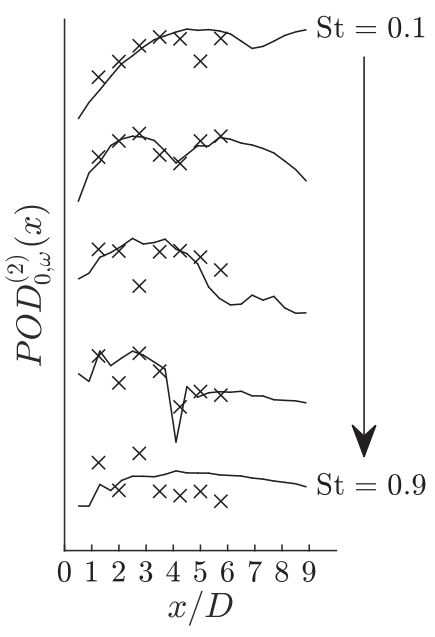

(b)

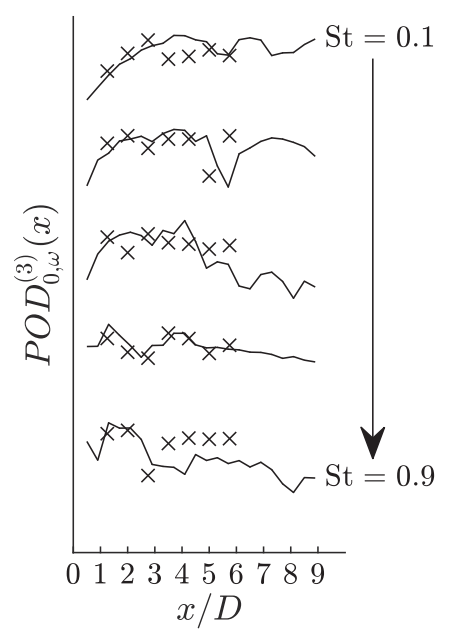

(c)

FIG. 12. $m=0$ POD modes for four-ring (_- ) and seven-ring $(\times$ and $\bigcirc)$ setups. In panels (b) and (c), every second St is skipped for clarity. Arbitrary scaling between St. (a) POD mode 1, (b) POD mode 2, and (c) POD mode 3 .

seven-ring and four-ring setups measure the same jets, the first several POD modes are the same and the seven-ring data can be projected onto the higher resolution basis. To determine $b_{m, \omega}^{(i)}$, the four-ring basis must be resampled at the same locations as the seven-ring data. Both the resampled basis and the original basis should be scaled by the same factor such that $\left|\xi_{m, \omega}^{(i)}(x)\right|=1$ for the resampled basis. This rescaling ensures that fluctuation energy is conserved-barring small errors-in the projection. In practice, since the seven-ring data have fewer degrees of freedom and POD modes higher than seven will be aliased into the lower modes, causing discrepancies in the modes obtained by four-ring and seven-ring arrays, this technique is only valid to the extent that POD modes obtained from the seven-ring array have the same shape as the four-ring array POD modes. Figure 12 shows that this is valid over the frequency range of interest for the first POD mode and that the validity is reasonable but deteriorating for the next two POD modes. These modes account for nearly all of the pressure fluctuation energy as indicated by Fig. 6. The agreement between the POD modes obtained using the four-ring and seven-ring arrays also helps to establish the convergence of POD, since both sets of modes are obtained from separate experiments.

Figure 13 shows an example of the reconstruction for $m=0$ using just the first POD mode in comparison with the full seven-ring signal. Also plotted is the far-field $\tilde{p}_{0}$ as a line plot in Fig. 13(a). The time scale is plotted as retarded time so that the far-field signal fluctuations are aligned with the fluctuations that are expected (by a first estimate) to have caused them. Retarded time is defined here as $\tau_{r}=\tau-\left|\vec{x}_{o}-\vec{x}_{s}\right| / c_{0}$. $\left(\left|\vec{x}_{o}-\vec{x}_{s}\right|\right.$ is the minimum distance between the ring of observer microphones and the circular cross section of the near-field cone at a given $x$ location. This is the travel distance for information from the near-field cone to the observer.) Here $\tau$ is measured from an arbitrary reference time within the signal. For the POD reconstruction in Fig. 13, the block size for the Fourier transform was $N=2440$ points $(\Delta \mathrm{St}=0.01)$.

In evaluating the effectiveness of the projection, the striking feature of the plots in Fig. 13 to consider is the similarity between Figs. 13(b) and 13(c). Visually, the first POD mode estimate captures all the salient dynamics of the full signal; however, we note that subtle effects leading to coherence decay are nonetheless absent. The difference is that the projection has a higher spatial resolution, and-as can be seen in Fig. 13(d) - it spans a greater spatial extent than the recorded signal, which is a direct result of the basis used in the projection. Within the span covered by the seven-ring basis, all the peaks and troughs of the signal align in the two plots and have the same 
DAVID E. S. BREAKEY et al.

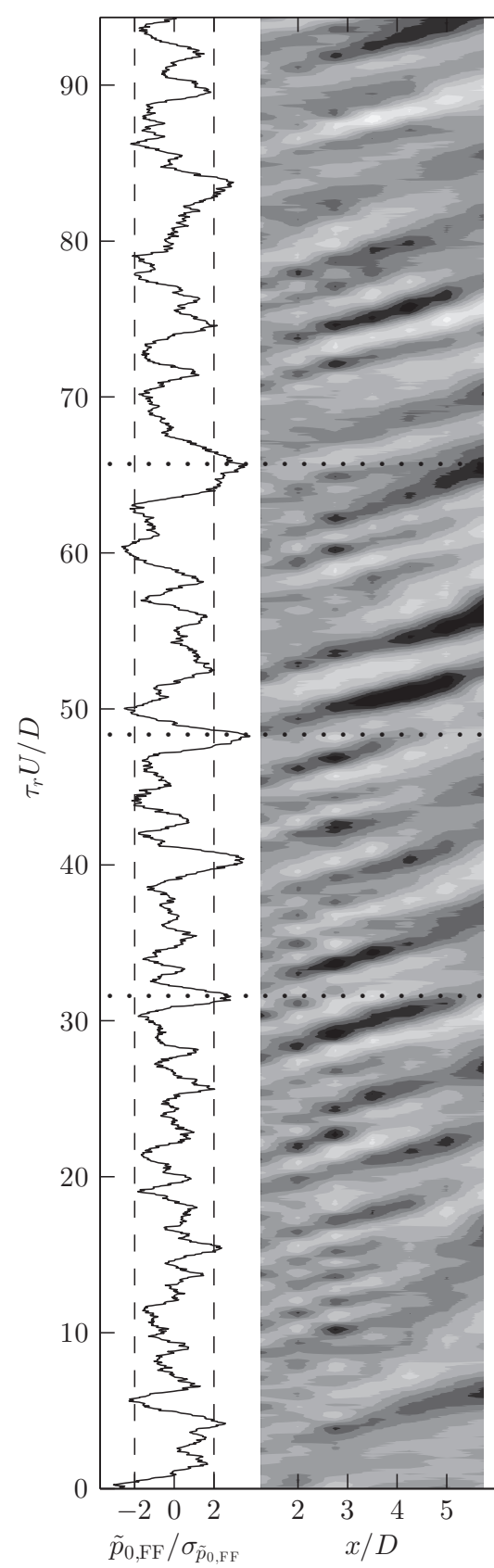

(a)

(b)

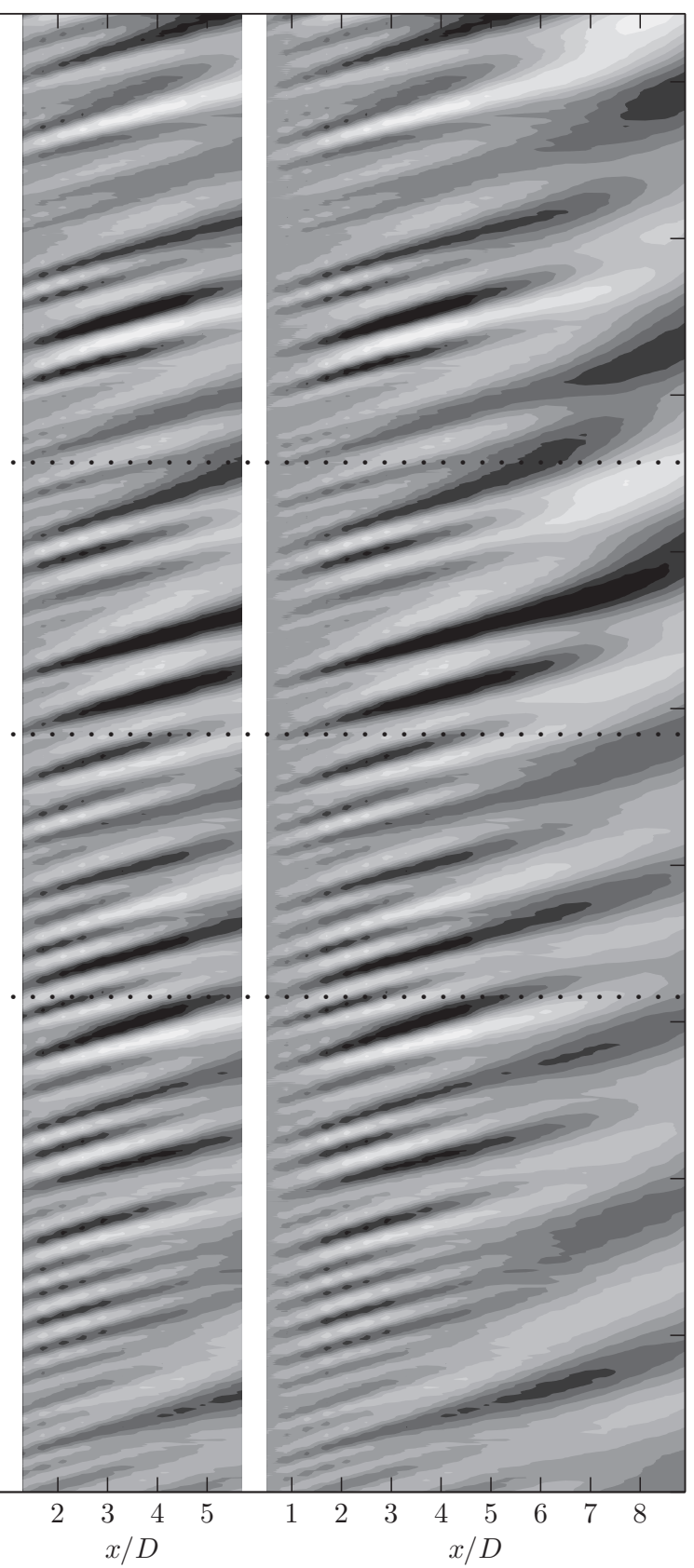

(d)

FIG. 13. Comparison between near-field and far-field $m=0$ pressure mode signals for both full signal and POD reconstruction. (a) Far-field signal plotted with $\pm 2 \sigma$ (dashed line) to show peaks, (b) full seven-ring near-field signal, (c) projection to POD mode 1 of the four-ring basis truncated to seven-ring axial extent, and (d) projection to POD mode 1 of the four-ring basis. The dotted horizontal lines highlight the alignment between selected far-field peaks and near-field features. Far-field pressure is plotted in retarded time. 


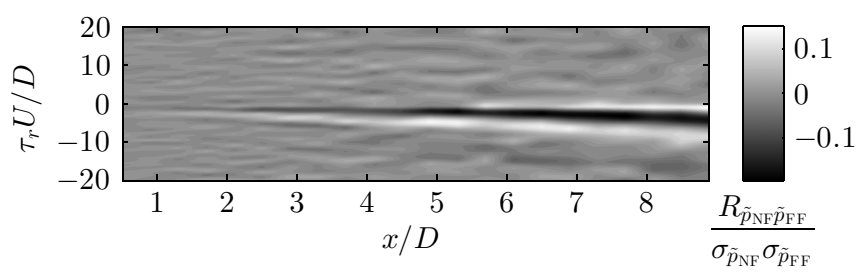

FIG. 14. Normalized retarded correlation between near-field $\tilde{p}_{0}$ and far-field $\tilde{p}_{0}$. The absolute maximum is $15.1 \%$.

magnitude. Statistically, the agreement between these two signals is expected to be as good as the agreement in Fig. 7 between the full signal and POD mode 1 curves. Knowing this, it is expected that beyond the end of the potential core, the POD mode 1 signal will deviate significantly from the full signal in the same manner that it does statistically in Fig. 7.

Beyond simply evaluating the projection technique, the results in Fig. 13 highlight the wave-packet structure of the near pressure field, which is not only present statistically-as previously shown by Suzuki and Colonius [30] and Tinney and Jordan [27]—but also in the time domain. This is clear from interpreting the features of the POD subplot. Clearly visible is a dominant feature that oscillates regularly and convects in the jet. The amplitude rises (growth region), levels out (saturation region), and decreases (decay region) beyond the potential core. This is the expected behavior of a wave packet.

\section{B. Near-field-far-field correlation}

As discussed in Sec. IC, near-field-far-field correlations help us understand the representations of the near field with respect to their significance to the far field, giving indications of the types of behavior captured by the POD modes. This is best understood by comparing full-signal correlations (Fig. 14) to correlations obtained between the reduced-order near-field representation and the full far-field signal (Figs. 15-18). Figure 14 shows the normalized cross-correlation- $-R_{\tilde{p}_{N F} \tilde{p}_{F F}} / \sigma_{\tilde{p}_{N F}} \sigma_{\tilde{p}_{F F}}$, where $\sigma_{a}$ is the root-mean-square of a time series $a(t)$-between the axisymmetric modes for the near-field and far-field signals $\left(\tilde{p}_{0}\right)$. As in Sec. V A, all the correlation plots shown here are plotted with respect to the retarded time lag, $\tau_{r}=\tau-\left|\vec{x}_{o}-\vec{x}_{s}\right| / c_{0}$, accounting for an estimated sound propagation time to the observation location. In the case of the correlations, $\tau$ is the correlation time tag. The extended nature of the peak signature indicates that the flow phenomena related to sound production are correlated over a large spatial extent [42]. If such a phenomenon also has high energy compared to other flow fluctuations, it should be detectable in the POD modes. The correlation between the reconstructed POD modes and the far field can indicate if POD efficiently captures the sound-producing phenomena. If the correlation for any particular mode is significantly higher than the baseline correlations in Fig. 14, then that mode is a good candidate for a sound generation mechanism and a possible target for control schemes. If the correlation magnitudes are similar across many POD modes, then this indicates more complex sound-producing wave-packet behavior. Since the first POD mode was shown in Sec. IV A to be nearly equivalent to the PSE prediction, the correlation between the first POD mode and the far field also constitutes a correlation between a time-domain projection of the near-field array onto the average linear wave-packet model and the far-field pressure.

With the current data, the correlation can be made directly with both the four-ring and seven-ring data for the full $\tilde{p}_{m}(x, t)$ for each azimuthal mode, but only the axisymmetric azimuthal mode is shown here. For the seven-ring data, the contributions of the POD modes to the near-field-far-field correlation are presented in Figs. 15 and 16 for the individual modes and the cumulative modes, respectively. Figure $16(\mathrm{~g})$ corresponds to the full correlation with $\tilde{p}_{0}(x, t)$, which is consistent with Fig. 14. For the four-ring data, this POD-mode-by-POD-mode correlation for all POD modes 
(a)

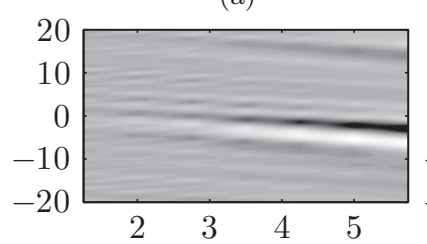

(d)

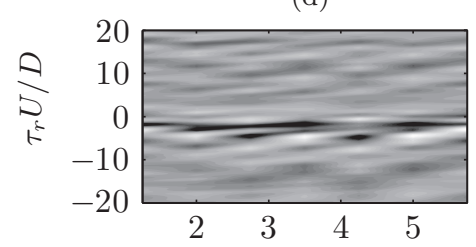

(g)

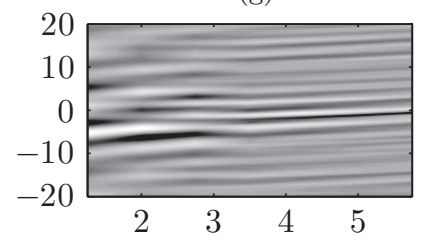

(b)

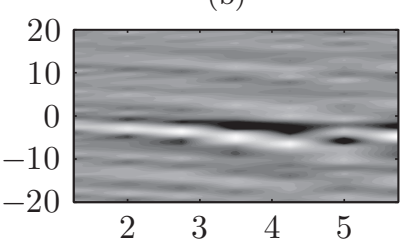

(e)

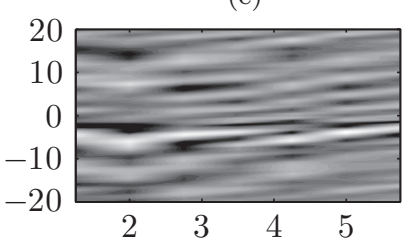

(c)

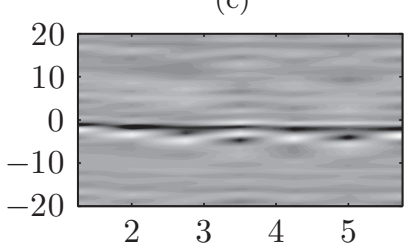

(f)

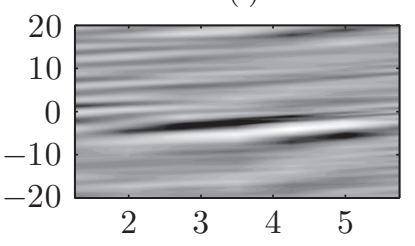

$$
x / D
$$

FIG. 15. Individual $m=0$ POD mode contributions to near-field-far-field correlation $\left(R_{\tilde{p}_{\mathrm{NF}} \tilde{p}_{\mathrm{FF}}} / \sigma_{\tilde{p}_{\mathrm{NF}}} \sigma_{\tilde{p}_{\mathrm{FF}}}\right)$ for 7-ring data. The absolute maximum for each subplot is indicated in brackets. (a) POD 1 (max. 13.7\%), (b) POD 2 (max. 13.5\%), (c) POD 3 (max. 14.0\%), (d) POD 4 (max. 9.7\%), (e) POD 5 (max. 8.7\%), (f) POD 6 (max. 8.4\%), and (g) POD 7 (max. 6.1\%).

is impossible because not all axial locations were recorded simultaneously. However, using the reconstruction method of Sec. V A, the seven-ring data were projected to the four-ring POD modes, allowing the higher resolution, longer axial extent correlations to be estimated. Figures 17 and 18 show these POD mode correlations both individually and cumulatively for the first three POD modes using this projection.

Figure 15(a) is nearly identical to the full correlation result plotted in Fig. 14, which shows that the first POD mode captures the salient correlation features. The interesting result here is that modes 2 and 3 do not show the expected behavior, which would be a nearly zero correlation with no physically important correlation features. In reality modes 2 and 3 also show similar correlation levels to the first mode, implying that they comprise acoustically important wave-packet dynamics. Furthermore, they are characterized by a predominantly convective space-time footprint. The higher order modes, on the other hand, show lower correlation levels and have a more complex space-time footprint, in some cases with peaks for instants other than the ones expected for propagation of acoustic waves. The fact that this correlation behavior is limited to the first three modes indicates that these modes together are capturing higher order behavior. There are fluctuations that could be said to be of low order (able to be captured with a few degrees of freedom) but not coherent in the traditional sense (periodic with unit coherence). These results are emphasized by Figs. 17 and 18 , in which, for the first three modes, a convective space-time signature is apparent as well as a similar maximum correlation level to the first POD mode. And in Fig. 15, the correlation maximum increases until POD 1-3, and then begins to decrease as more POD modes are added, which is the same result that would be expected if uncorrelated noise were to be added to the near-field signal. These results make it apparent that higher order fluctuations are present as proposed in Sec. V C.

As discussed earlier, recent work has established a link between POD modes and stochastically forced linear wave packets. In the so-called resolvent framework, for linear instability waves subject to "external" stochastic forcing, the POD modes are identical to the singular resolvent modes if 
(a)

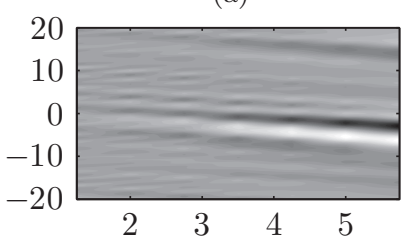

(d)

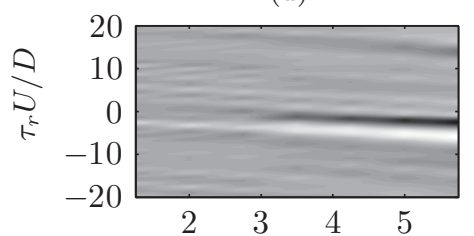

(g)

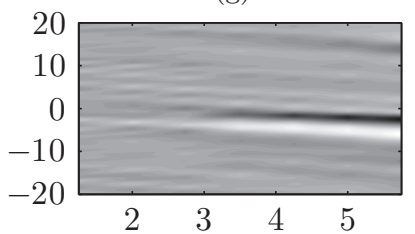

(b)

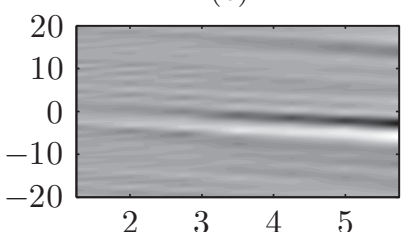

(e)

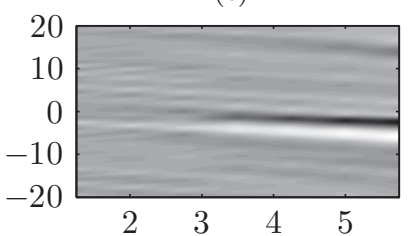

(c)

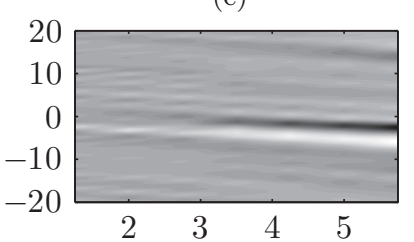

(f)

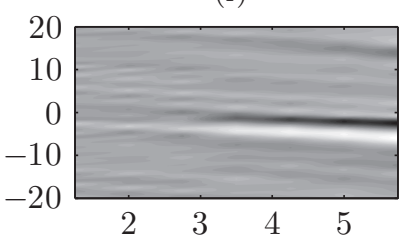

$$
x / D
$$

FIG. 16. Cumulative $m=0$ POD mode near-field-far-field correlations $\left(R_{\tilde{p}_{\mathrm{NF}} \tilde{p}_{\mathrm{FF}}} / \sigma_{\tilde{p}_{\mathrm{NF}}} \sigma_{\tilde{p}_{\mathrm{FF}}}\right)$ for 7 -ring data. The absolute maximum for each subplot is indicated in brackets. (a) POD 1-1 (max. 13.7\%), (b) POD 1-2 (max. 16.5\%), (c) POD 1-3 (max. 17.2\%), (d) POD 1-4 (max. 16.4\%), (e) POD 1-5 (max. 16.0\%), (f) POD 1-6 (max. $15.9 \%)$, and $(\mathrm{g})$ POD $1-7(\max .15 .8 \%)$.

the forcing is given by white noise. The resolvent response modes form an orthonormal basis that can be used to expand the data in terms of a hierarchy of optimally forced linear-wave structures. The first mode comprises the optimal response, the highest gain structure, while the suboptimals permit the description of more complex forced-wave behavior. In this context, we find thus that suboptimal modes remain relevant for sound radiation, which was also observed by Jeun et al. [28] in a resolvent analysis of subsonic jets. The fact that three POD modes reproduce the downstream amplitude envelope, the overall coherence envelope (known to be closely tied to acoustic efficiency) and are now found to characterize a significant part of the near-field-far-field correlation that is underpinned by hydrodynamic near-field activity, supports the idea that these modes have captured the acoustically important wave-packet dynamics.

To understand the implications of these results on the far-field sound emissions, it is necessary to consider the emissions related to these low-order structures with the observed far-field sound. This is

(a)

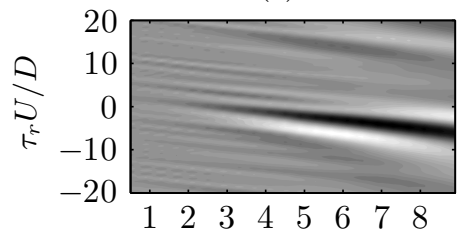

(b)

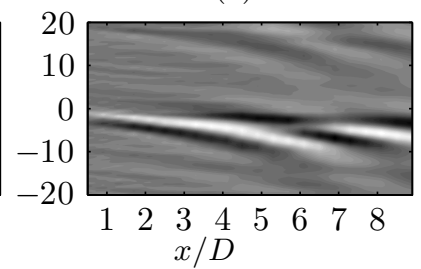

(c)

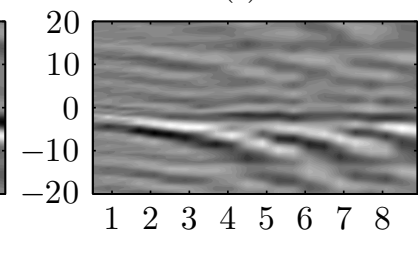

FIG. 17. Individual $m=0$ POD mode contributions to near-field-far-field correlation $\left(R_{\tilde{p}_{\mathrm{NF}} \tilde{p}_{\mathrm{FF}}} / \sigma_{\tilde{p}_{\mathrm{NF}}} \sigma_{\tilde{p}_{\mathrm{FF}}}\right)$ for the reduced-order projection using Eq. (8). The absolute maximum for each subplot is indicated in brackets. (a) POD 1 (max. 15.1\%), (b) POD 2 (max. 14.0\%), and (c) POD 3 (max. 14.2\%). 
(a)

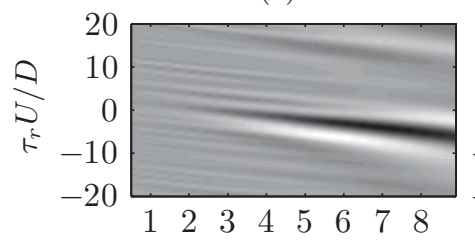

(b)

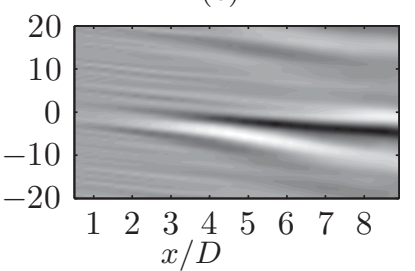

(c)

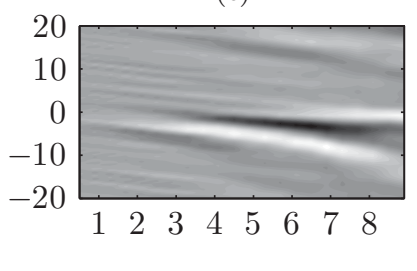

FIG. 18. Cumulative $m=0$ POD mode near-field-far-field correlations $\left(R_{\tilde{p}_{\mathrm{NF}} \tilde{p}_{\mathrm{FF}}} / \sigma_{\tilde{p}_{\mathrm{NF}}} \sigma_{\tilde{p}_{\mathrm{FF}}}\right)$ for the reducedorder projection using Eq. (8). The absolute maximum for each subplot is indicated in brackets. (a) POD 1-1 (max. 15.1\%), (b) POD 1-2 (max. 15.1\%), and (c) POD 1-3 (max. 17.0\%).

done by using established techniques for projecting the near-field activity to the far field statistically and can also be done in the time domain. The aim here is primarily to determine the effect of these features on the efficiency of the wave-packet emissions.

\section{Far-field predictions from near-field wave packets}

\section{Formulation for statistical predictions}

Reba et al. [31,43] showed, by changing coordinates to a spherical system $(\mathcal{R}, \Theta, \varphi)$ centered at the virtual origin of the conical array, that it is possible to write the far-acoustic field due to a pressure distribution on the near-field conical surface as

$$
P_{m, \omega}\left(\mathcal{R}_{o}, \Theta_{o}\right)=2 \pi \sin \alpha \int_{0}^{\infty} P_{m, \omega}(\mathcal{R}, \alpha) \frac{\partial g_{m, \omega}}{\partial \Theta}\left(\mathcal{R}, \alpha ; \mathcal{R}_{o}, \Theta_{o}\right) d \mathcal{R},
$$

where $g_{m, \omega}$ is a tailored Green's function for the reduced wave equation and the subscript $o$ indicates the observer coordinate location. Reba et al. [31,43] continued with Eq. (9) to write the autospectral density of the far-field pressure as

$$
\begin{aligned}
& \left\langle P_{m, \omega}\left(\mathcal{R}_{o}, \Theta_{o}\right) P_{m, \omega}^{*}\left(\mathcal{R}_{o}, \Theta_{o}\right)\right\rangle \\
& \quad=4 \pi^{2} \sin ^{2} \alpha \iint_{0}^{\infty} \mathrm{R}_{m, \omega}\left(\mathcal{R}_{1}, \mathcal{R}_{2}\right) \frac{\partial g_{m, \omega}}{\partial \Theta}\left(\mathcal{R}_{1}, \alpha ; \mathcal{R}_{o}, \Theta_{o}\right) \frac{\partial g_{m, \omega}^{*}}{\partial \Theta}\left(\mathcal{R}_{2}, \alpha ; \mathcal{R}_{o}, \Theta_{o}\right) d \mathcal{R}_{1} d \mathcal{R}_{2},
\end{aligned}
$$

which gives the far-field sound pressure level (SPL). A brief validation of this statistical prediction technique is presented in Appendix B alongside a validation of the time-domain prediction technique introduced below. Their predictions fit well with experimental data for high-speed $(M \approx 1.6)$ jets up to about $\theta<45^{\circ}$ at $\mathrm{St}=0.25$, but the results were significantly worse for slower jets $(M \approx 0.9)$ and higher frequency $(\mathrm{St} \geqslant 0.40)$. A possible explanation for this error is that error introduced by making predictions from an array covering only a small amount of the jet's axial extent (an issue considered in more detail in Sec. V C 3). This error is also greater at lower Mach number.

\section{Formulation for time-domain predictions}

Though the statistical prediction technique has been used before, to the best of our knowledge, a prediction of the time-domain far-field pressure has not been attempted. Taking the inverse Fourier transform of Eq. (9) gives

$$
\tilde{p}_{m}\left(\mathcal{R}_{o}, \Theta_{o}, t\right)=\int P_{m, \omega}\left(\mathcal{R}_{o}, \Theta_{o}\right) e^{\mathrm{i} \omega t} d \omega,
$$

which is the time-resolved far-field pressure corresponding to the azimuthal mode $m$. In conjunction with the POD mode projection of Sec. V A, this technique can give time-domain far-field predictions from near-field microphone measurements. To implement this with discrete data, Eq. (9) must first be solved for every frequency for the number of fast Fourier transform (FFT) points, $N$, used to 


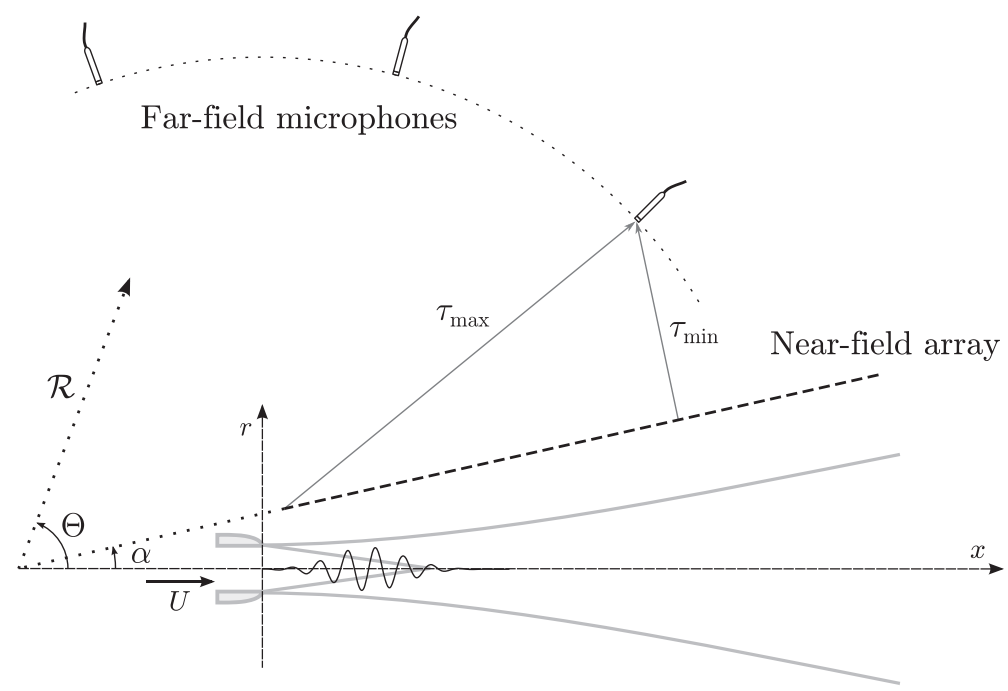

FIG. 19. Geometric considerations for time-domain predictions with a finite-window Fourier transform.

calculate $P_{m, \omega}\left(\mathcal{R}_{o}, \Theta_{o}\right)$, which allows the inverse FFT (IFFT) to be applied. In practice, for each far-field observation location, the signal at each time step is dependent on a number of time steps in the near field. Based on causality, since the fluid everywhere outside of the array cone is quiescent, the contribution from each near-field location takes $\tau=\left|\vec{x}_{o}-\vec{x}_{s}\right| / c_{0}$ to arrive, making each far-field point dependent on a duration of data equal to $\tau_{\max }-\tau_{\min }$, where $\tau_{\min }$ and $\tau_{\max }$ are the minimum and maximum propagation times, respectively, from locations on the array with significant contribution to the far field. So in practice, the number of valid time-series points is limited by the physical layout of the array and far-field observer because the discontinuity at the edge of the FFT window causes an error in the far-field prediction. Since the propagation distances are finite (see Fig. 19) and the sound speed is constant in the ambient fluid, the number of affected points is limited, and the number of valid points can be estimated by

$$
N_{\text {Valid }}=N-\left(\tau_{\max }-\tau_{\min }\right) f_{s} .
$$

When calculating a long time series, the series can be broken into blocks, and overlapping the blocks by at least $N-N_{\text {Valid }}$ points overcomes the issue of corrupted points. A validation of the procedure and details of the implementation are presented in Appendix B.

\section{Experimental limitations to far-field prediction}

A key difficulty that has been identified during past attempts to make far-field predictions from near-field experimental arrays is the fact that any experimental array only covers a finite portion of the source domain $[44,45]$. In the current setup, the near-field array's axial extent is limited to $0.5 \leqslant x / D \leqslant 8.9$, so no information is recorded about the decay of the pressure fluctuations beyond this extent. Though it would seem reasonable to assume that once the pressure fluctuations decay to a relatively small value like is observed here, it would not be necessary to capture them, this does not seem to be the case. The best explanation for this is that the truncation introduces a spurious, highly efficient sound source at the end of the array. This effect has been noted by others and discussed in detail [45]. A more thorough investigation of the spurious source caused by truncation of the experimental array for this particular case has been presented by Breakey [46]. In that work, even attempts to use simple models for the decay with increasing $x$ to mitigate the spurious source were not fruitful, even in a model problem used for validation. 


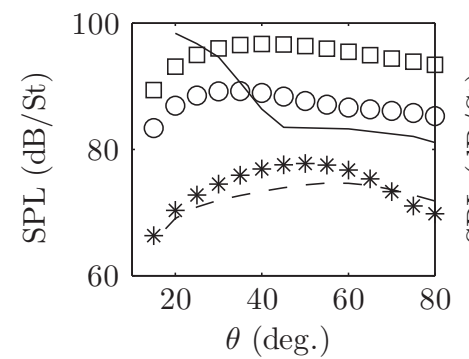

(a)

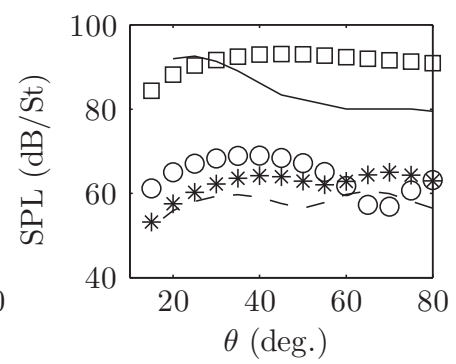

(b)

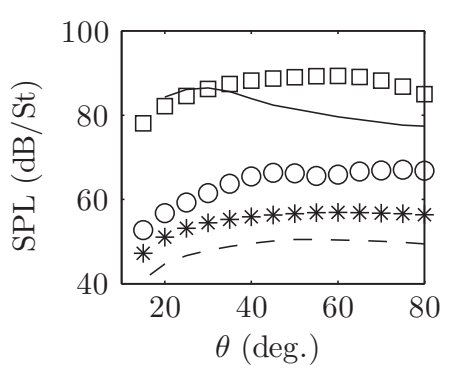

(c)

FIG. 20. Comparison of Green's-function-predicted directivities from the current four-ring measurements for the $M=0.6$ jet. $\square$, Full prediction; $\bigcirc$, POD mode 1 prediction; - - -, PSE prediction; $*$, truncated PSE prediction $(0.5 \leqslant x / D \leqslant 8.9)$; and - , Cavalieri et al.'s [2] experimental data. (a) $\mathrm{St}=0.2$, (b) $\mathrm{St}=0.5$, and (c) $\mathrm{St}=0.8$.

In Reba et al. [31,43], with an array of similar extent, predictions matched experimental data well for higher Mach numbers $(M \approx 1.6)$, but were much worse for lower speed jets $(M \approx 0.9)$. This seems to match well with the conclusions of Wang et al. [47], which indicate that the error increases significantly as Mach number decreases. However, in the model problem tested for validation of this spurious source effect for the current configuration, it is clear that the effect has a much greater influence at low and high $\theta$ and that the directivity resulting from the prediction technique should still have a similar overall amplitude even if it has a different shape [46]. As it is this overall level that we are primarily interested in in what follows, notwithstanding the obvious effect of this source, we pursue application of the technique to the experimental data.

\section{Application to experimental data}

The directivity results for the four-ring data are compared to the data measured previously for this jet by Cavalieri et al. [2] in Fig. 20. Clearly, the Green's-function-predicted directivity is inaccurate. As discussed in Sec. VC 3, the effect of the axial truncation of the near-field array is believed to underpin this error. To estimate this effect, the PSE CSM was truncated to $0.5 \leqslant x / D \leqslant 8.9$, and the result is also presented in Fig. 20. The truncation does cause a significant deviation in the predicted directivity shape but not the overall level. It is possible that this truncation is the main reason for the difference in the shape of the directivity, but this is currently impossible to verify since attempts to model the decay of the wave packet at larger $x$ have not been fruitful [46]. Nevertheless, the truncation effect does not seem to explain the significant difference in overall magnitude across all angles.

It is notable that across all frequencies the full-data prediction is closest in terms of magnitude. Using just the PSE or first POD mode reduces the prediction by as much as $20 \mathrm{~dB}$ over all angles, which indicates that the source of the large discrepancy is likely associated with mechanics missing from the PSE model as opposed to simple truncation errors. Similar discrepancies, noted by Baqui et al. [26] while using homogeneous linear models to compute hydrodynamic wave packets, have been attributed to the inability of these models to capture coherence decay. Baqui et al. [26] show how, using the two-point coherence measured in the present work, those linear models can be corrected such that they recover the correct acoustic efficiency. In light of these results, the large differences in level observed, between the acoustic extrapolation of the full data on one hand, and that of the linear models and the first POD mode on the other, underline once again the importance of the two-point coherence for the acoustic efficiency of wave packets. The close reproduction of the coherence envelope that is achieved with three POD modes (in comparison with the unit coherence associated with the first mode alone) again illustrates how the second and third modes carry the signature of the structures associated with jitter and coherence decay.

The acoustic importance of these first few modes can be surmised without performing a full far-field projection by performing a spatial Fourier transform on the CSM and plotting the result 


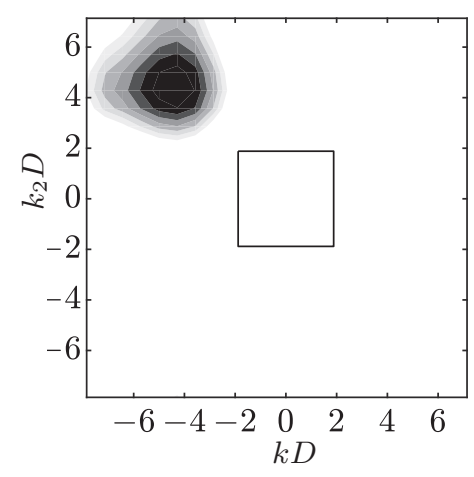

(a)

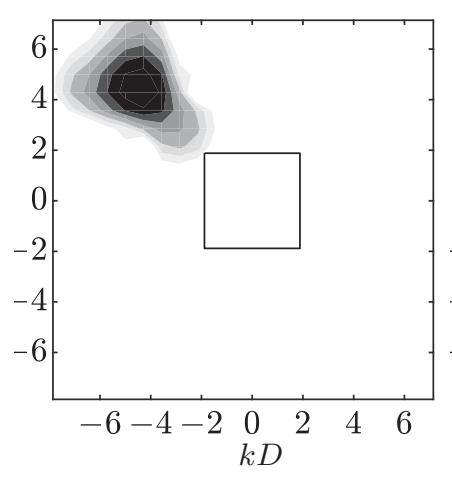

(b)

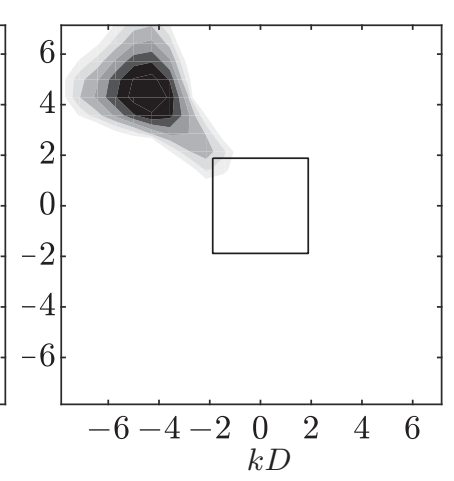

(c)

FIG. 21. CSM plots for $\mathrm{St}=0.5$ plotted by wave number (obtained by spatial Fourier transform). The central square section, delimited by $k= \pm k_{\infty}$ and $k_{2}= \pm k_{\infty}$, where $k_{\infty}=\omega / c_{0}$ encloses radiation that "leaks" to the far field. Contour levels are logarithmic. (a) POD 1-1, (b) POD 1-2, and (c) POD 1-3.

as a function of wave number, $k$. This result is plotted for each combination of POD modes in Fig. 21. As was discussed in Sec. IB, and as presented by Cavalieri and Agarwal [11], only energy that falls within $k<k_{\infty}$, where $k_{\infty}=\omega / c_{0}$ is the acoustic wave number, radiates to the far field. Graphically, this is energy that falls within the central squares in Fig. 21. As can be seen here, as the extra POD modes are incorporated, more energy falls within this square, giving evidence that these spatiotemporal "jitter" effects are important in far-field predictions.

\section{CONCLUSION}

In recent years, wave-packet models for jets have shown considerable promise for the prediction of acoustically important flow fluctuations, particularly for fluctuations that are azimuthally and axially coherent, often associated with low-angle sound emissions. Recent analysis of the far-field sound emissions and the in-flow velocity field of a subsonic jet by Cavalieri et al. [12] found close agreement between wave-packet predictions based on PSE and experimental fluctuation measurements on the jet centerline up until the end of the potential core, indicating the presence of wave packets at least up to this point. The agreement was found to break down downstream, and from their measurements, the mechanism responsible was unclear. Baqui et al. [26] also noted disagreement in the two-point coherence.

The current investigation, based on the same jet, indicates that linear wave packets persist in the jet beyond the end of the potential core, with a clear signature in the first POD mode. However, the full autospectra amplitudes in the downstream region (i.e., without POD filtering) are significantly higher than predictions from wave-packet models. Including more than one POD mode reduces this discrepancy. In the coherence envelope, this is equivalent to allowing extra degrees of freedom, which we show to capture most of the experimental coherence features while maintaining a low-order representation. Interpreting these results is possible by considering the relationship between POD and the linear operator proposed by Farrell and Ioannou [35], reworked in the frequency domain by Semeraro et al. [25]. For both amplitude and coherence, a small number of POD modes, interpreted physically as high-gain wave packets that arise in response to forcing by turbulent nonlinearities, are sufficient to provide low-order reconstructions of these wave-packet statistics.

A complicating factor observed in the present data is that the PSE predictions suffer from a damping of the acoustic waves relative to the primary wavelength of the near-field wave packet, but this damping does not significantly affect the near-field wave-packet shape. Further, it is clear that the fundamental difference between prediction and experiment is the inability of PSE to capture the higher order structure of the wave packet, which leads ultimately to additional, significant far-field discrepancies. 
A time-domain reconstruction of the near-field fluctuations for each POD mode has been performed, allowing the near-field fluctuations to be projected onto an empirically obtained wave packet (POD mode). This made it possible to consider the correlation between the POD modes and the far-field sound. The correlation indicated that the first POD mode captures the salient features of the space-time correlation to the far-field sound, but the higher POD modes also showed significant correlations to the far field, indicating that a single POD mode is insufficient to extract all the acoustically important flow fluctuations. The POD-resolvent link again allows physical interpretation of these results: Both the optimal and suboptimal high-gain, forced wave packets play a role in sound radiation, a result in line with the recent findings of Jeun et al. [28].

The use of a statistical far-field prediction based on a Green's function gave poor directivity predictions. It was found that acoustic extrapolation using either the homogeneous PSE solution or a single POD mode leads to global levels that are as much as $30 \mathrm{~dB}$ lower than the experiment. On the other hand, when the full near-field data are used, despite a spurious source effect related to the finite extent of the near-field array and consistent with other investigations, globally correct levels are recovered. A frequency-wave-number transform of the near-field cross-spectral density matrix, considered using different numbers of POD modes, shows that this considerable change in acoustic efficiency is due, as suggested by the near-field-far-field correlations, to the crucial role played by the higher order POD modes. When a single mode is used, the fluctuation energy lies away from the radiating wave numbers, whereas when three POD modes are included, the spectral peak is found to stretch into the radiating region of wave-number space. Given the relationship between the POD modes and the highest-gain resolvent modes, this result illustrates how the acoustic efficiency of the wave packets is underpinned by forcing by background turbulence, which activates higher order degrees of freedom that produce jitter, coherence decay, and the observed acoustic efficiency.

\section{ACKNOWLEDGMENTS}

D.E.S.B. thanks Dr. Craig Meskell for his discussions concerning the analysis of the data. Science Foundation Ireland supported this work under Contract No. 09/RFP/ENM2469. D.E.S.B. was also supported by the Natural Sciences and Engineering Research Council of Canada. This work was partially supported through the EU-Russian program ORINOCO (FP7-AAT-2010-RTD-Russia; Project No. 266103) and by the Agence Nationale de la Recherche (ANR) Project No. ANR-12BS09-0024 through the project Cool Jazz (COntrol-Oriented Linear and nonlinear models for Jet Aeroacoustic ZZ).

\section{APPENDIX A: VERIFICATION OF PSE UNDERPREDICTION}

Section IV D describes the evidence that the factor of 10 first mentioned in Sec. IV A is a physical rather than erroneous value. To confirm this, an independent dataset was also examined. The result is a strong indication that this effect is physical and is related to PSE overestimating the radial decay rate of the wave packets in the pressure field.

The data used for this comparison are the the first POD mode of the LES computation of the same jet considered in this study but operating at $M=0.9$ by Brès et al. [38] (POD modes computed by Ref. [20]) and the PSE predictions for this configuration computed by Cavalieri et al. [48]. Again, the jet has a diameter of $D=50 \mathrm{~mm}$, but here $\mathrm{Re}=1 \times 10^{6}$. The comparison of these results within the jet flow and at low $r$ have already been presented by Brès et al. [38].

Figure 22 shows the axial velocity and pressure PSE wave-packet estimates compared to the first POD mode LES data at the locations of the experimental array. The scaling constant was obtained by forcing the velocity result to match the PSE at $x / D=0.25$ and $r / D=0.4$ and applied to both the velocity and pressure PSE predictions. In Fig. 22(b), the PSE clearly underestimates the LES fluctuations, and the magnitude of this underestimation is higher for higher $r_{A}$. So the issue is that PSE overestimates the radial decay rate of the pressure fluctuations. Since the factor is not as high as 10 for this data, it seems that the effect is weaker at higher Mach number. This also explains why 


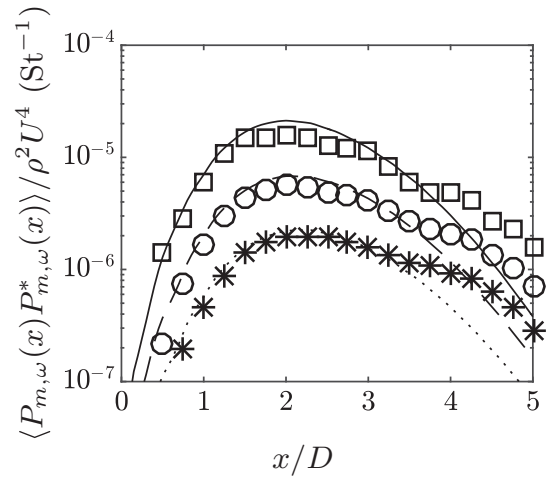

(a)

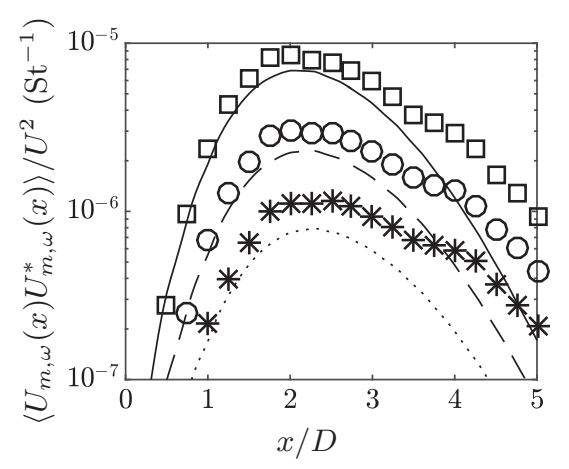

(b)

FIG. 22. Comparison of PSE estimates (lines; from Ref. [48]) and first POD mode of LES (symbols; from Ref. [20], every fifth data point shown) for $m=0, M=0.9$, and $\mathrm{St}=0.61$ using the array locations from the current seven-ring experiment. — and $\square, r_{A} / D=0.6 ;---$ and $\bigcirc, r_{A} / D=0.7 ; \ldots \ldots$ and $*, r_{A} / D=0.8$. (a) Velocity and (b) Pressure.

Schmidt et al. [20] found no discrepancy between pressure PSE and LES values in their comparisons: All of their comparisons were made at low $r$, where this effect is not noticeable.

The reason for this effect can be inferred from Fig. 23, which shows the LES and PSE results up to high $r$. The POD mode from the LES data is very close to the prediction for lower $r$, but as $r$ increases, first the deviation appears and eventually the magnitude flattens completely. This can be attributed to the dominance of the acoustic fluctuations as $r$ increases. Since PSE cannot accurately predict the acoustic fluctuations radiating from subsonic jets, it underpredicts the fluctuation magnitude, even with an array as close as $r_{A} / D=0.6$ in the $M=0.6$ case.

\section{APPENDIX B: FAR-FIELD PREDICTION VALIDATION}

The code developed for the calculation of the far-field pressure has been extensively validated by Léon [49] and Léon and Brazier [29], and a modified validation suitable for time-domain signals is presented here. The validation problem is generated for the azimuthal pressure mode by prescribing

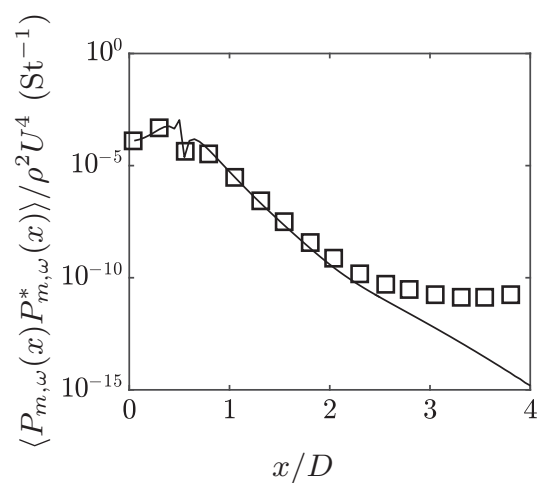

(a)

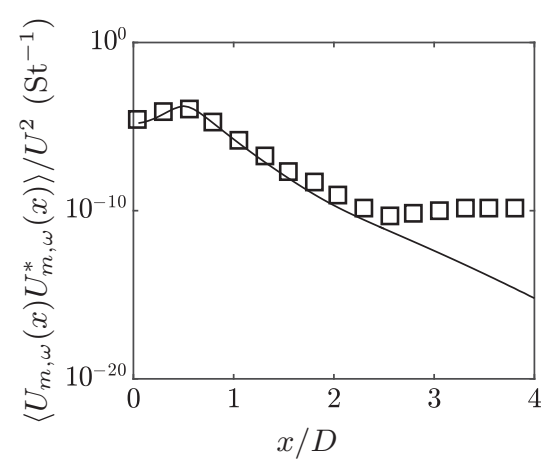

(b)

FIG. 23. Radial comparison of PSE estimates (lines, from Ref. [48]) and first POD mode of LES (symbols; from Ref. [20]) for $m=0, M=0.9$, and $\mathrm{St}=0.61$ at $x / D=2$. (a) Velocity and (b) Pressure. 
DAVID E. S. BREAKEY et al.

TABLE II. Model constants used in validation problem.

\begin{tabular}{lccc}
\hline \hline$a$ & $b$ & $c$ & $d$ \\
\hline 1.04 & $2.47 D$ & 0.15 & $1.20 D$ \\
\hline \hline
\end{tabular}

a simple line source for the Lighthill stress tensor given by

$$
T_{11}(x, t)=A(x) \tilde{p}_{\exp }\left(t-x / U_{c}\right),
$$

where $A(x)$ represents an arbitrary spatial envelope, $U_{c}=0.6 U$ is an estimated wave-packet convection velocity, and $\tilde{p}_{\exp }(t)$ is an experimental pressure signal taken from the azimuthal mode of the microphone ring at $x / D=4.1$. This was chosen so that the frequency content of the validation problem would be similar to the real case. $A(x)$ was taken to correspond roughly to the amplitude envelope of the first four-ring POD mode using the model function

$$
A(x)=a \exp \left[-\frac{(x-b)^{2}}{[c(x-b)+d]^{2}}\right],
$$

where the coefficients $a, b, c$, and $d$ were determined from a least-squares fit of the experimental data. For reference, the coefficients are tabulated in Table II.

The Lighthill integral for this line source is solved using a high spatiotemporal-resolution numerical calculation given by

$$
p^{\prime}\left(\vec{x}_{o}, t\right)=\frac{1}{4 \pi} \int \frac{1}{r} \frac{\partial^{2} T_{i j}\left(\vec{x}_{s}, t-r / c_{0}\right)}{\partial x_{i} \partial x_{j}} d \vec{x}_{s},
$$

where the subscript $o$ again indicates the observer location. The integral is solved for observers at positions along the near-field array from the array origin $\left(X_{0}\right)$ to $x / D=70$ with a spacing of $0.4 D$ as well as at the position of each far-field microphone $\left(\left|\vec{x}_{o}\right| / D=47.1\right.$ and $\left.15^{\circ} \leqslant \theta \leqslant 80^{\circ}\right)$. To avoid edge effects from the finite time window, the Lighthill solution is calculated for a larger time window

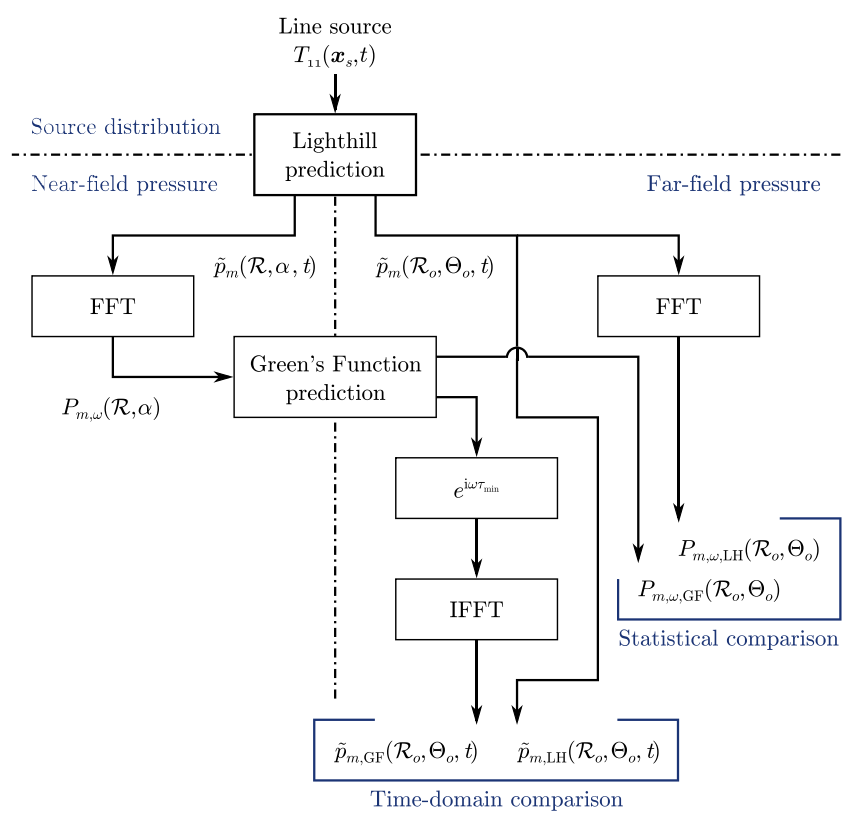

FIG. 24. Analysis procedure for time-domain far-field signal prediction. 


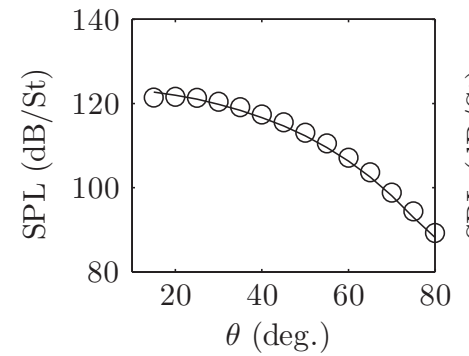

(a)

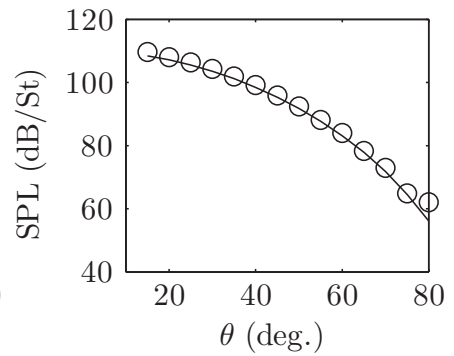

(b)

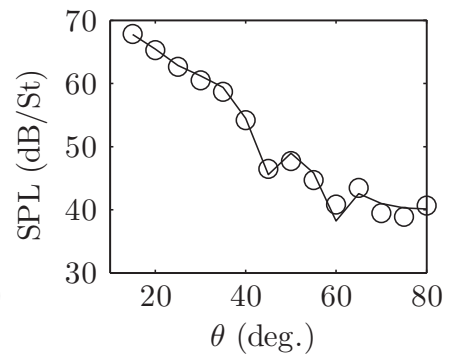

(c)

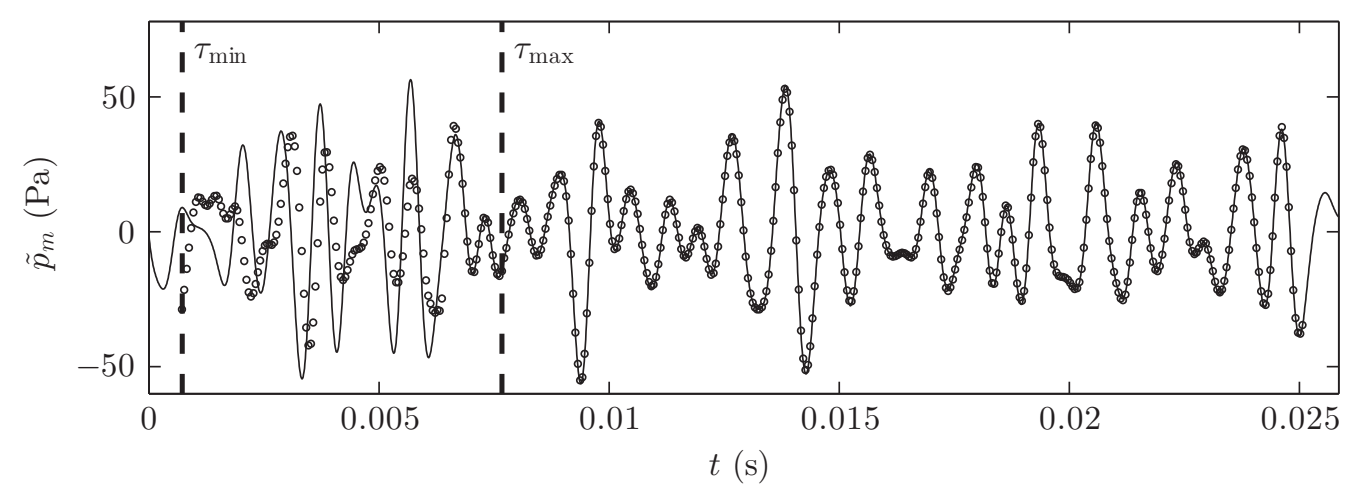

(d)

FIG. 25. Validation results for the far-field Green's function predictions from the near-field array with $X_{0} \leqslant x / D \leqslant 70$. - , Lighthill solution; $\bigcirc$, Green's function solution. (a) Statistical prediction $(\mathrm{St}=0.2)$, (b) Statistical prediction $(\mathrm{St}=0.5)$, (c) Statistical prediction $(\mathrm{St}=0.8)$, and (d) Time-domain prediction at $\theta=20^{\circ}$ (Every fifth data point of the Green's function result is shown.).

and only the central points are used in the predictions. The Green's function prediction for the far field is made by propagating the near-field Lighthill solution from Eq. (B2) using the Green's function technique. This prediction is then compared directly to far-field Lighthill solution calculated directly from Eq. (B2). The entire procedure is presented schematically in Fig. 24, where the subscripts LH and GF represent the solutions by the Lighthill and Green's function methods, respectively.

In a direct numerical implementation of the time-domain formulation, the discontinuity also appears somewhere in the middle of the time series, and the periodicity of the Fourier transform causes some of the information from the end of the time series to be shifted to the front. This causes the portion of the data occurring before $\tau_{\min }$ to be aligned improperly. The proper alignment can easily be recovered-along with the shifting of the discontinuity to the beginning of the time series-by applying a frequency-domain phase shift before performing the IFFT. This operation is represented in the schematic in Fig. 24 by the operator $e^{\mathrm{i} \omega \tau_{\min }}$. Alternatively, the erroneous points could be placed at the end of the signal by replacing $\tau_{\min }$ with $\tau_{\max }$ in the operation.

Figure 25 shows the results for both the statistical prediction and the time-domain prediction. Both sets of results used blocks of $N=2440$ points corresponding to $\Delta \mathrm{St}=0.01$, where the time-domain result was calculated from a single block, and the statistical results were calculated by a series of blocks with $50 \%$ overlap. Since the statistical results compare only single frequencies, a Hann window was used in the calculation of $\mathrm{R}_{m, \omega}$ to avoid spectral leakage. No windowing was used for the time-domain result. The Green's function predictions faithfully reproduce the Lighthill solution in both cases, verifying the propagation technique. In the time-domain case, the result shown is one block of $N=2440$ points, and the points between $\tau_{\min }$ and $\tau_{\max }$ are corrupted as predicted. 
[1] P. Jordan and T. Colonius, Wave packets and turbulent jet noise, Annu. Rev. Fluid Mech. 45, 173 (2013).

[2] A. V. G. Cavalieri, P. Jordan, T. Colonius, and Y. Gervais, Axisymmetric superdirectivity in subsonic jets, J. Fluid Mech. 704, 388 (2012).

[3] E. Mollo-Christensen, Measurements of near field pressure of subsonic jets, Tech. Rep. ASRL TR 1009, Massachusetts Institute of Technology, Aeroelastic and Structures Research Laboratory, Cambridge, MA, 1963 (unpublished).

[4] E. Mollo-Christensen, Jet noise and shear flow instability seen from an experimenter's viewpoint, ASME J. Appl. Mech. 34, 1 (1967).

[5] S. C. Crow and F. H. Champagne, Orderly structure in jet turbulence, J. Fluid Mech. 48, 547 (1971).

[6] D. G. Crighton, Basic principles of aerodynamic noise generation, Prog. Aerosp. Sci. 16, 31 (1975).

[7] S. C. Crow, Acoustic gain of a turbulent jet, in Annual Meeting of the Division of Fluid Dynamics, American Physical Society (American Physical Society, Washington, DC, 1972).

[8] D. G. Crighton and P. Huerre, Shear-layer pressure fluctuations and superdirective acoustic sources, J. Fluid Mech. 220, 355 (1990).

[9] N. D. Sandham, C. L. Morfey, and Z. W. Hu, Sound radiation from exponentially growing and decaying surface waves, J. Sound Vib. 294, 355 (2006).

[10] A. V. G. Cavalieri, P. Jordan, A. Agarwal, and Y. Gervais, Jittering wave-packet models for subsonic jet noise, J. Sound Vib. 330, 4474 (2011).

[11] A. V. G. Cavalieri and A. Agarwal, Coherence decay and its impact on sound radiation by wave packets, J. Fluid Mech. 748, 399 (2014).

[12] A. V. G. Cavalieri, D. Rodrguez, P. Jordan, T. Colonius, and Y. Gervais, Wave packets in the velocity field of turbulent jets, J. Fluid Mech. 730, 559 (2013).

[13] F. Kerhervé, P. Jordan, A. V. G. Cavalieri, J. Delville, C. Bogey, and D. Juvé, Educing the source mechanism associated with downstream radiation in subsonic jets, J. Fluid Mech. 710, 606 (2012).

[14] A. Michalke, Instabilität eines kompressiblen runden Freistrahls unter Berücksichtigung des Einflusses der Strahlgrenzschichtdicke, Z. Flugwiss. 19, 319 (1971); Instability of a compressible circular free jet with consideration of the influence of the jet boundary layer thickness, NASA TM 75190, NASA.

[15] D. G. Crighton and M. Gaster, Stability of slowly diverging jet flow, J. Fluid Mech. 77, 397 (1976).

[16] T. Herbert, Parabolized stability equations, Annu. Rev. Fluid Mech. 29, 245 (1997).

[17] K. Gudmundsson and T. Colonius, Instability wave models for the near-field fluctuations of turbulent jets, J. Fluid Mech. 689, 97 (2011).

[18] J. W. Nichols and S. K. Lele, Global modes and transient response of a cold supersonic jet, J. Fluid Mech. 669, 225 (2011).

[19] L. Lesshafft, P. Huerre, and P. Sagaut, Aerodynamic sound generation by global modes in hot jets, J. Fluid Mech. 647, 473 (2010).

[20] O. Schmidt, A. Towne, T. Colonius, P. Jordan, V. Jaunet, A. V. Cavalieri, and G. A. Brès, Super- and multi-directive acoustic radiation by linear global modes of a turbulent jet, in Proceedings of the 22nd AIAA/CEAS Aeroacoustics Conference (AIAA, Reston, VA, 2016), paper AIAA 2016-2808.

[21] S. Beneddine, D. Sipp, A. Arnault, J. Dandois, and L. Lesshafft, Conditions for validity of mean flow stability analysis, J. Fluid Mech. 798, 485 (2016).

[22] B. J. McKeon and A. S. Sharma, A critical-layer framework for turbulent pipe flow, J. Fluid Mech. 658, 336 (2010).

[23] G. Tissot, M. Zhang, F. C. Lajs, A. V. G. Cavalieri, and P. Jordan, Sensitivity of wave packets in jets to nonlinear effects: The role of the critical layer, J. Fluid Mech. 811, 95 (2017).

[24] A. Towne, T. Colonius, P. Jordan, A. V. Cavalieri, and G. A. Brès, Stochastic and nonlinear forcing of wave packets in a Mach 0.9 jet, in Proceedings of the 21st AIAA/CEAS Aeroacoustics Conference, AIAA AVIATION Forum (AIAA, Reston, VA, 2015), paper AIAA 2015-2217.

[25] O. Semeraro, V. Jaunet, P. Jordan, A. V. G. Cavalieri, and L. Lesshafft, Stochastic and harmonic optimal forcing in subsonic jets, in Proceedings of the 22nd AIAA/CEAS Aeroacoustics Conference (AIAA, Reston, VA, 2016), paper AIAA 2016-2935.

[26] Y. B. Baqui, A. Agarwal, A. V. G. Cavalieri, and S. Sinayoko, A coherence-matched linear source mechanism for subsonic jet noise, J. Fluid Mech. 776, 235 (2015). 
[27] C. E. Tinney and P. Jordan, The near pressure field of co-axial subsonic jets, J. Fluid Mech. 611, 175 (2008).

[28] J. Jeun, J. W. Nichols, and M. R. Jovanovi, Input-output analysis of high-speed axisymmetric isothermal jet noise, Phys. Fluids 28, 047101 (2016).

[29] O. Léon and J.-P. Brazier, Investigation of the near and far pressure fields of dual-stream jets using an Euler-based PSE model, in Proceedings of the 19th AIAA/CEAS Aeroacoustics Conference (AIAA, Reston, VA, 2013), paper AIAA 2013-2280.

[30] T. Suzuki and T. Colonius, Instability waves in a subsonic round jet detected using a near-field phased microphone array, J. Fluid Mech. 565, 197 (2006).

[31] R. Reba, S. Narayanan, and T. Colonius, Wave-packet models for large-scale mixing noise, Int. J. Aeroacoust. 9, 533 (2010).

[32] D. Jung, S. Gamard, and W. K. George, Downstream evolution of the most energetic modes in a turbulent axisymmetric jet at high Reynolds number, part 1: The near-field region, J. Fluid Mech. 514, 173 (2004).

[33] G. Berkooz, P. Holmes, and J. L. Lumley, The proper orthogonal decomposition in the analysis of turbulent flows, Annu. Rev. Fluid Mech. 25, 539 (1993).

[34] J. L. Lumley, The structure of inhomogeneous turbulent flows, in Atmospheric Turbulence and Radio Propagation, edited by A. M. Yaglom and V. I. Tatarski (Nauka Publishing House, Moscow, Russia, 1967), pp. 166-178.

[35] B. F. Farrell and P. J. Ioannou, Stochastic forcing of the linearized Navier-Stokes equations, Phys. Fluids A: Fluid Dyn. 5, 2600 (1993).

[36] G. Dergham, D. Sipp, and J.-C. Robinet, Stochastic dynamics and model reduction of amplifier flows: The backward facing step flow, J. Fluid Mech. 719, 406 (2013).

[37] A. Towne, G. A. Brès, and S. K. Lele, A statistical jet-noise model based on the resolvent framework, in Proceedings of the 23rd AIAA/CEAS Aeroacoustics Conference, AIAA AVIATION Forum (AIAA, Reston, VA, 2017), paper AIAA 2017-3706.

[38] G. A. Brès, V. Jaunet, M. Le Rallic, P. Jordan, T. Colonius, and S. K. Lele, Large eddy simulation for jet noise: The importance of getting the boundary layer right, in Proceedings of the 21st AIAA/CEAS Aeroacoustics Conference, AIAA AVIATION Forum (AIAA, Reston, VA, 2015), paper AIAA 2015-2535.

[39] A. Sinha, D. Rodríguez, G. A. Brès, and T. Colonius, Wave packet models for supersonic jet noise, J. Fluid Mech. 742, 71 (2014).

[40] A. Towne and T. Colonius, One-way spatial integration of hyperbolic equations, J. Comp. Phys. 300, 844 (2015).

[41] M. Zhang, P. Jordan, G. Lehnasch, A. V. G. Cavalieri, and A. Agarwal, Just enough jitter for jet noise? in Proceedings of the 20th AIAA/CEAS Aeroacoustics Conference, AIAA AVIATION Forum (AIAA, Reston, VA, 2014), paper AIAA 2014-3061.

[42] D. E. S. Breakey, J. A. Fitzpatrick, and C. Meskell, Aeroacoustic source analysis using time-resolved PIV in a free jet, Exp. Fluids 54, 1 (2013).

[43] R. Reba, J. Simonich, and R. Schlinker, Chevron nozzle effects on wavepacket sources in a supersonic jet, in Proceedings of the 18th AIAA/CEAS Aeroacoustics Conference (33rd AIAA Aeroacoustics Conference) (AIAA, Reston, VA, 2012), paper AIAA 2012-2253.

[44] T. Colonius and S. Lele, Computational aeroacoustics: Progress on nonlinear problems of sound generation, Prog. Aerosp. Sci. 40, 345 (2004).

[45] P. Martínez-Lera and C. Schram, Correction techniques for the truncation of the source field in acoustic analogies, J. Acoust. Soc. Am. 124, 3421 (2008).

[46] David E. S. Breakey, Time-resolved noise source analysis in subsonic turbulent jets, Ph.D. thesis, University of Dublin-Trinity College, Dublin, Ireland, 2014.

[47] M. Wang, J. B. Freund, and S. K. Lele, Computational prediction of flow-generated sound, Annu. Rev. Fluid Mech. 38, 483 (2006).

[48] K. Sasaki, A. V. G. Cavalieri, P. Jordan, O. T. Schmidt, T. Colonius, and G. A. Brès, High-frequency wavepackets in turbulent jets, J. Fluid Mech. 830, R2 (2017).

[49] O. Léon, Étude du rayonnement acoustique des instabilités hydrodynamiques de jets double-flux par les équations de stabilité parabolisées (PSE), Ph.D. thesis, University of Toulouse, Toulouse, France, 2012 [Acoustics of hydrodynamic instabilities in dual-stream jets using parabolized stability equations (PSE)]. 